\title{
INVESTIGACIÓN
}

Recibido: 06/03/2019 --- Aceptado: 29/04/2019 --- Publicado: 15/03/2020

\section{ACOSO ESCOLAR, VIOLACIÓN Y SUICIDIO EN TWITTER: SEGUNDA TEMPORADA DE «POR TRECE RAZONES»}

\author{
Bullying, rape and suicide on Twitter: second season of 13 Reasons Why
}

Víctor Manuel Pérez-Martínez ${ }^{1}$ : Universidad San Jorge. España. vmperez@usj.es

Beatriz Aparicio Vinacua: Universidad de Zaragoza. España. beatriz6av@gmail.com

María Dolores Rodríguez-González: UNEXPO, Venezuela. mariadrodriguezdp@gmail.com

\section{RESUMEN}

Tanto si son ficción como si incorporan un formato documental, las series para adolescentes (teens series) pueden convertirse en una referencia para los adolescentes. La serie 13 Reasons Why (Por trece razones), en su segunda temporada, generó interés entre los adolescentes, las familias y el sector educativo. El objetivo general de esta investigación es analizar cuantitativamente los comentarios sobre el acoso escolar, las violaciones y los suicidios en Twitter vinculados con la segunda temporada de 13 Reasons Why. Metodología: se concretó en un análisis de contenido de los tuits que contenían las etiquetas (hashtag) \#13ReasonsWhy y/o \#PorTreceRazones. Resultados y discusión: temas complejos como la violación, el suicidio, la relación padre-hijo, el acoso y el ciberacoso se plantearon en torno al suicidio de Hannah Baker. Conclusiones: Una de las conclusiones del estudio establece que 13 Reasons Why promovió el debate sobre el acoso escolar, la violación y el suicidio siendo Twitter un canal donde se exponen temas tan complejos para los adolescentes.

PALABRAS CLAVE: Por trece razones - Hannah Baker - Twitter - Acoso escolar y series juveniles - Suicidio y series juveniles - Violación y series juveniles - Netflix

\footnotetext{
1 Víctor Manuel Pérez-Martínez: Doctor en Ciencias de la Información. Profesor en la Universidad San Jorge, España. Fue investigador principal: «Cultura Digital: mediaciones y discursos», «Cyberspace Working Group» (con reconocimiento del Gobierno de Aragón).
} 
Pérez-Martínez, V. M., Aparicio Vinacua, B. y Rodríguez-González, M. D.

Acoso escolar, violación y suicidio en twitter: segunda temporada de «por trece razones».

\begin{abstract}
Whether they are fiction or incorporate a documentary format, the teen series can become a reference for teenagers. The series 13 Reasons Why, in its second season, generated interest among adolescents, families and the education sector. The general objective of this research is to quantitatively analyse the comments on school bullying, rape and suicide on Twitter linked to the second season of 13 Reasons Why. Methodology: It was specified in a content analysis of the tweets that contained the hashtag \#13ReasonsWhy and/or the hashtag \#PorTreceRazones. Results and discussion: Complex issues such as rape, suicide, the parent-child relationship, bullying and cyber-bullying were raised around Hannah Baker's suicide. Conclusions: One of the conclusions of the study states that 13 Reasons Why promotes the debate on school bullying, rape and suicide being Twitter a channel where such complex issues are exposed for adolescents.
\end{abstract}

KEY WORDS: 13 Reasons Why - Hannah Baker - Twitter - Bullying and teen series Suicide and teen series - Rape and teen serie - Netflix

\title{
ASSÉDIO MORAL ESCOLAR, ESTUPRO E SUICÍDIO NO TWITTER: SEGUNDA TEMPORADA DE «13 REASONS WHY»
}

\section{RESUMO}

Tanto se são ficção como se incorporam um formato documental, as séries para adolescentes (teens series) tornam-se uma referência para os adolescentes. A série 13 Reasons Why (Por treze razões), na sua segunda temporada, gerou interesse entre os adolescentes, as famílias e o setor educativo.O objetivo geral desta pesquisa é analisar quantitativamente os comentários sobre o assédio moral escolar, os estupros e os suicídios no Twitter vinculados com a segunda temporada de 13 Reasons Why. Metodología: se concretou em uma análise de conteúdo dos tweets que continham as etiquetas (hashtag) \#13ReasonsWhy y/o \#PorTreceRazones. Resultados e discussão: temas complexos como o estupro, o suicídio, a relação pai-filho, o assédio e o ciberbullying se fizeram ao redor do suicídio de Hannah Baker. Conclusões: Uma das conclusões do estudo estabelece que 13 Reasons Why promoveu o debate sobre o assédio moral, o estupro e o suicidio sendo Twitter um canal onde se expõe temas tão complexos para os adolescentes.

PALAVRAS CHAVE: Por treze razões - Hannah Baker - Twitter - Assédio escolar e séries juvenis - Suicídio e séries juvenis - Estupro e séries juvenis - Netflix 
Pérez-Martínez, V. M., Aparicio Vinacua, B. y Rodríguez-González, M. D. Acoso escolar, violación y suicidio en twitter: segunda temporada de "por trece razones».

\section{Como citar el artículo:}

Pérez-Martínez, V. M., Aparicio Vinacua, B. y Rodríguez-González, M. D. (2020). Acoso escolar, violación y suicidio en twitter: segunda temporada de "por trece razones». [Bullying, rape and suicide on Twitter: second season of 13 Reasons Why].Vivat Academia. Revista de Comunicación, 153, 137-168. doi: https://doi.org/10.15178/va.2020.153.137-168 Recuperado de http://www.vivatacademia.net/index.php/vivat/article/view/1220

\section{INTRODUCCIÓN}

Son frecuentes las investigaciones académicas sobre cómo las redes sociales facilitan a los adolescentes y a los jóvenes crear entornos virtuales. En estos espacios virtuales las audiencias juveniles comentan temas complejos a partir de la visualización de series juveniles (teen series). En algunos de estos estudios se profundiza sobre la comunicación mediada a través de las tecnologías de la información y cómo contribuye al conocimiento de las nuevas narrativas audiovisuales. Se analiza también el uso de las redes sociales como fuentes de información y canales de comunicación en los adolescentes (Nabawy, Moawad, Gad, y Ebrahem, 2016; Gangopadhyay y Dhar, 2014) o cómo está vinculada esta participación a etapas vitales (Sprada Barbosa et al., 2018; Benassini Félix, 2018; Aubrey, Behm-Morawitz, y Kim, 2014a; Damme, 2010).

En este artículo la investigación se focaliza en la segunda temporada de 13 Reasons Why, en España se tradujo Por trece razones. Una ficción seriada, creada por y para la plataforma Netflix, que generó un amplio debate sobre el acoso escolar, la violación y el suicidio. Reflexiones que se trasladaron a redes sociales como Twitter.

\subsection{Adolescente y ficción seriada.}

Las teen series son un «amplio abanico de manifestaciones televisivas mundiales consideradas para adolescentes por su contenido, su target, su contexto de programación o sus datos demográficos de recepción» (Raya, Sanchez-Labella, y Duran, 2018). No son novedosas (García-Muñoz y Fedele, 2011; Guarinos, 2009; Morduchowicz, 2008; Pasquier, 1997) y han sido estudiadas desde diversas perspectivas (Vázquez-Herrero et al., 2019; Escalas, 2017; Tapia Frade y Martín Guerra, 2016; Josefa y Heras, 2016; Rios, 2015; Del Moral Pérez y Villalustre Martínez, 2015; Aubrey, Behm-Morawitz, y Kim, 2014b; Whiteside, Hardin, Decarvalho, Carillo, y Smith, 2013; García García, 2011; Damme y Bauwel, 2010; Feasey, 2006).

La novedad son los estudios que exploran, en las teen series, como la tecnología digital y online, la cual evoluciona vertiginosamente, contribuye a los cambios de conductas o de apropiación de estas audiencias de la narrativa audiovisual. Series como One Tree Hill (2003-2012) Gossip Girl (2007-2012), The Vampire Diaries (20092017), Riverdale (2017), 90210 (2008-2013) o Euphoria (2019) son algunas de las series que han captado el interés del público adolescente/joven y del mundo académico 
Pérez-Martínez, V. M., Aparicio Vinacua, B. y Rodríguez-González, M. D. Acoso escolar, violación y suicidio en twitter: segunda temporada de «por trece razones».

(Becattini, 2018; Ryalls, 2016; Fradegradi (2016); Santandreu Aranda, 2015; Langue, 2011; Van Damme, 2010; García-Muñoz y Fedele, 2011; Palin, 2009; Guarinos, 2009).

Las audiencias asumen pactos implícitos de lectura que transforman los mundos de la ficción en realidad (García-Noblejas, 2017). Los fans de una teen serie son un tipo de espectador que se sumerge en la ficción y a la vez participa e interactúa en los entornos online aportando sus valoraciones sobre aquello que experimenta viendo la serie. El ciberespacio se convierte en un ecosistema compartido con otros para comentar estas experiencias: sea por la temática, el eufemístico anonimato, por los perfiles que interactúan o por el hecho de expresar una opinión o información concreta. Sin necesidad de que la persona tenga que exponer su identidad en internet (Gerrad, 2017). Lo importante está en ser parte de la red.

Las actividades en internet «están vinculadas a la creación de significado» (Tortajada y Willem, 2019, p. 101). En las ficciones seriadas (Carrión Domínguez, 2019) hay que incorporar las interpretaciones y apropiaciones que realizan las audiencias sobre esos contenidos. Adicionalmente, al margen de los cambios que la tecnología y la industria audiovisual están aportando en la concepción de las producciones audiovisuales y en sus modelos de difusión y de negocio, el entorno online se puede concebir también como «un espacio para la construcción social de las identidades y de las comunidades» (Tortajada y Willem, p. 101).

La audiencia se apropia de la narración, la interpreta, aporta significados y reconstruye los significados en el contexto de la interactividad que facilitan las comunidades virtuales. Otra cuestión es la profundidad del debate durante el tiempo que estas comunidades estén activas en la red. Serán los temas, subtemas, personajes o la identificación de las realidades individuales de las audiencias con la ficción, el argumento para visualizar series específicas (Aubrey et al., 2014b; Navarro-Abal y Climent-Rodríguez, 2014; Navarro-Abal, Climent-Rodríguez y Fernández-Garrido, 2012; Figuera-Maz, Tortajada y Araüna, 2008). Se puede plantear la existencia de un determinado grado de engagement que la audiencia puede alcanzar y mantener en relación con las ficciones seriadas.

\subsection{La audiencia desde la perspectiva de la narrativa transmedia}

Esta capacidad o potencialidad de la audiencia de asumir con mayor énfasis, frecuencia o profundidad una postura activa en los entornos online es una de las vías para la expansión de las historias que plantea la narrativa transmedia (Jenkins, 2010). Teniendo en cuenta el contexto online actual -movilidad, redes sociales, hiperconectividad o la amplia diversidad de plataformas interactivas- el reto está en cómo gestiona la audiencia sus opiniones y en cuáles comunidades virtuales o canales son compartidas.

Según el tipo de ficción se configura un perfil de audiencia específica. Carrión Domínguez (2019) se refiere a las series Qualitity TV como «una tipología concreta de series, que se inicia en la década de los ochenta y que explota a finales de siglo, hasta 
Pérez-Martínez, V. M., Aparicio Vinacua, B. y Rodríguez-González, M. D. Acoso escolar, violación y suicidio en twitter: segunda temporada de «por trece razones».

llegar a nuestros días convertida en un fenómeno sociocultural inimaginable hace cuarenta años» (p. 113). Series como Hill Street Blues (1981) o ER (1994). Carrión Domínguez actualiza los criterios que Robert J. Thompson utilizó para el análisis de la Qualitity $T V$. Seleccionamos las siguientes aportaciones relacionadas con la audiencias: a) de una audiencia sofisticada y elitista, gracias a la tecnología y a otra visión del modelo de negocio, en la actualidad, «el público al que se dirigen, en general, es mucho más amplio» (p. 116); b) de una audiencia reducida se ha alcanzado, en algunas series, audiencias masivas por tres factores: fidelidad de la audiencia (Fringe), calidad (The Wire) y el reconocimiento de la industria (Mad Men) (p. 116); c) «sus tramas buscan la controversia, tienen una implicación social» (p. 117).

En los nuevos tipos de audiencia en el ciberespacio, y concretamente en propuestas transmedia como El Ministerio del Tiempo, Scolari y Establés parten de las ideas de Hill: centran el análisis en los fans, «no solo como posibles creadores profesionales de productos mediáticos, sino también en su papel a la hora de cuestionar las mediciones de las audiencias de series, que pueden ser consideradas cult TV». Es decir, «cuentan con un público especializado y minoritario» (Scolari y Establés, 2017, p. 1014).

Las formas como las audiencias se expresan evolucionan y se segmentan dejando atrás las características tradicionales de la televisión; en este marco audiovisual de referencia, los jóvenes asumen el consumo audiovisual y su participación, como audiencia, con otros criterios: «Se interesan por nuevas experiencias transmedia que aúnen los diferentes prismas en los que discurre su rutina como son las redes sociales, la web o la gamificación y que se favorezca su capacidad productora de mensajes» (Vázquez-Herrero, González-Neira, y Quintas-Froufe, 2019, p. 75). En síntesis, las integran a su cotidianidad (Rodríguez Illera, Martínez Olmo, y Galván, 2019; Fuenmayor y García Aranguren, 2018).

Las series no se visualizan como antes. Era usual tener que esperar unos días o unas semanas para visualizar los episodios. El acceso actualmente es factible a todos los episodios creando la necesidad, ansiedad u oportunidad, de visualizarlos cuanto antes para comentarlos con otros seguidores de la serie. Comentarios que se trasladan a las redes sociales $u$ otros espacios en línea.

Desde la perspectiva de una propuesta narrativa multiplataforma, en la cual el espectador produce contenidos a partir de la experiencia con el producto audiovisual, hay resultados que ponen en valor la concepción de las producciones audiovisuales como unas unidades narrativas que transcienden el discurso audiovisual siendo la propia audiencia quienes generan esa «propagabilidad» (García Pastor, 2018). En gran medida porque productores y directores consideran a los fans y a la audiencia en general como partícipes de las historias. La narrativa audiovisual adopta formas aún por definirse y los límites entre las narrativas transmedia, crossmedia, multiplataforma o cualquier otra que pudiera surgir generan problemas metodológicos para su estudio. 
Pérez-Martínez, V. M., Aparicio Vinacua, B. y Rodríguez-González, M. D. Acoso escolar, violación y suicidio en twitter: segunda temporada de «por trece razones».

Antonié Vallet (1977) fue visionario al señalar que en la transformación que vivía la sociedad había un interrogante fundamental: «¿cuál es el lenguaje del hombre, de la sociedad de hoy?» (Vallet, p. 9). Su planteamiento fundamental con respecto al «lenguaje total» es que era un «lenguaje verbo - audio - visual» (p. 18). La evolución de la tecnología y de las narrativas hacen más complejo ese «lenguaje total». En esta línea se evidencia la necesidad de formar a los jóvenes en la lectura y comprensión de los nuevos códigos. Incorporar estos temas en la enseñanza (Sáiz Serrano y Parra Monserrat, 2017; Sánchez Escámez y Baena Sánchez, 2016; Aierbe-Barandiaran y Oregui-González, 2016; Llorent y Marín, 2013; Aguaded Gómez, 2005).

La narrativa transmedia contribuye a un planteamiento renovado de la narrativa audiovisual y en la cual los elementos de la ficción se dispersan, de una manera sistemática, a través de múltiples canales con la finalidad de «crear una experiencia de entretenimiento unificada y coordinada»(Jenkins, 2010, p. 944). Al margen de estas características específicas, las experiencias transmedia está dirigida a la audiencia interesada en la historia que se cuenta; «a una figura muy concreta: la del fan» (García Pastor, 2018, p. 71). Esta forma de comprender las historias influye en otros contextos creativos estableciendo formas diferentes de contar esas historias (Trillo-Domínguez, Del Moral, y Sedeño-Valdellós, 2019; Sidorenko Bautista, Calvo Rubio y Cantero de Julián, 2018; Julián Gonzales, 2018).

Henry Jenkins plantea evidencias de una generación transmedia que asume con normalidad las claves de una cultura participativa que aporta su propia visión de la sociedad en convivencia con su cultura personal: «In countries across the globe, they combine the local traditions of popular culture with the forms of digital expression now 142elevisió globally in ways that previous generations could not have imagined»(Jenkins, marzo 2010). El contexto transmedia requiere una participación colaborativa y activa para expandir la historia entre los usuarios que interactúan en los espacios digitales, característica bien recibida por los adolescentes y jóvenes en una sociedad en la cual la interconectividad es una demanda constante.

Se plantea una alfabetización acorde con la realidad mutimodal de la actualidad. La alfabetización, concebida como la acción de 'alfabetizar', «enseñar a alguien a leer o a escribir», está desfasada: «If traditional literacy was book-centred or, in the case of media literacy, mostly 142elevisión-centred, then multimodal literacy places digital networks and interactive media experiences at the centre of its analytical and practical experience» (Scolari, 2018, p. 15).

Scolari aporta evidencias de la importancia que tiene para los adolescentes y jóvenes la capacidad de producir, intercambiar y consumir contenidos; $\mathrm{y}$, todo ello, en el marco de un ciberespacio globalizado y accesible a través de las plataformas móviles: «The Transmedia Literacy team confirmed the existence of a broad spectrum of situations, skills, strategies, content production/sharing/consumption processes and alternative uses of media. Transmedia skills represent a diverse and uneven topography» (Scolari, 2018, p. 18). En este escenario, las redes sociales son canales que contribuyen a ampliar la experiencia transmedia y poner de alguna 
Pérez-Martínez, V. M., Aparicio Vinacua, B. y Rodríguez-González, M. D. Acoso escolar, violación y suicidio en twitter: segunda temporada de «por trece razones».

manera en valor la participación de los jóvenes en internet. Incluso surge un «nuevo producto cultural derivado» a partir de dos o más mundo de ficción: crossovers (Guerrero-Pico y Scolari, 2016, p. 187).

En esta línea, los datos indican que los jóvenes encuentran en las redes sociales un espacio para el encuentro: «El hilo conductor de nuestras prácticas sociales es la narrativa y su forma de discurso predominante es lo que denominamos transmedia» (Fuente Prieto, Lacasa Díaz y Martínez-Borda, 2019, p. 176). Las redes sociales son espacios potenciales de participación como lo evidencia las investigaciones sobre el comportamiento de la audiencia y de los fans en redes sociales (Cortés Gómez, Martínez Borda y De la Fuente Prieto, 2016) (Lacalle y Castro, 2018), Facebook (López y Fernández, 2016) (Gómez Rubio y López Vidaldes, 2015) (Tur-Viñes y Rodríguez Ferrándiz, 2014), YouTube (Sánchez-Olmos, 2016) (Feijoo y Pavez, 2019); Twitter (Donstrup, 2019) u otras plataformas con comunidades online de fans (Zhang y Cassany, 2019).

\subsubsection{Reasons Why}

13 Reasons Why generó expectativa en adolescentes, familias y centros educativos. La novela original de Jay Asher (2007), orientada a un público joven-adulto, la adaptó Brian Yorkey a formato audiovisual para Netflix y su primera temporada, estrenada el 31 de marzo de 2017, tuvo repercusión en el contexto mediático (Bertolli Filho y Pontalti Monari, 2018; Carmichael y Whitley, 2018), en la blogósfera (Wulandari, Shinta Hapsari y Bram, 2018) y fue referente entre los adolescentes y jóvenes. La segunda temporada, no relacionada directamente con la novela de Jay Asher, profundizó en las historias de los personajes y las razones/explicaciones por las cuáles actuaron en la primera temporada. En la tercera temporada, disponible desde el 23 de agosto de 2019, la trama se aleja del hilo conductor de las dos temporadas anteriores.

Un componente clave del éxito de la serie fue ser una serie original pensada para el modelo de negocio y de distribución de Netflix. Una empresa proveedora de vídeos por demanda (video-on-demand services), con presencia en prácticamente todos los países y, en algunas ocasiones, la plataforma con el mayor tráfico de internet generado en este entorno digital por sus características técnicas (Böttger, Cuadrado, Tyson, Castro, y Uhlig, 2018). Esta experticia adquirida en la distribución de vídeo por demanda y en la producción de series de televisión captó el interés de un número significativo de suscriptores.

\subsubsection{Primera temporada}

El argumento principal de la primera temporada son las trece razones que impulsaron a Hannah Baker (Katherine Langford) al suicidio. Esta historia es relatada en primera persona por Hannah Baker en 13 casetes (cassette, tape) que envió, antes de su suicidio, a uno de sus compañeros de clase, Clay Jensen (Dylan Minnette), para que los escuchara y los enviara a las otras personas que aparecían 
Pérez-Martínez, V. M., Aparicio Vinacua, B. y Rodríguez-González, M. D. Acoso escolar, violación y suicidio en twitter: segunda temporada de «por trece razones».

mencionadas en los casetes. Dos reglas planteadas por Hannah: escuchar y pasar los casetes a la siguiente persona. Una advertencia, si las reglas se rompen hay una copia de esos casetes y se divulgarán sus contenidos sin reparar en las consecuencias que tendrían para las personas involucradas. En el episodio del suicidio el método utilizado por Hannah fue cortarse las venas de las muñecas en la bañera de su casa: fue una escena explícita visualmente. Diferente a la versión de la novela escrita por Jay Asher en la cual la opción que tomaría Hannah sería el consumo de pastillas.

La violación, el suicidio, la relación padres-hijos o el ciberacoso fueron temáticas abordadas en los episodios. Esta primera temporada no pasó desapercibida por la comunidad científica y se publicaron estudios desde varias disciplinas (Wulandari, Shinta Hapsari y Bram, 2018). Investigaciones relacionadas con: el perfil de los personajes adolescentes (Raya, Sánchez-Labella y Durán, 2018); la perspectiva jurídica (Abreu Sancho y Huamán Cruz, 2018), el rol que deben desempeñar los responsables docentes en los colegios (Oliveira y Ribeiro, 2018). Los temas claves fueron: el acoso escolar (Prawiyadi, Irawan Aritonang y Wijayanti, 2018) y el suicidio (Renato Nardi y Feliciano Brigagão, 2018; Atarama-Rojas y Requena Zapata, 2018; Cambra Badii y Mastandrea, 2017).

Al respecto, Netflix, ante las polémicas surgidas por esta temporada emitió un episodio especial Beyond the Reasons Season 1 (posteriormente emitiría Beyond the Reasons Season 2) para abordar con expertos de diversas áreas las pautas para crear debates a partir de los episodios de 13 Reasons Why. Netflix promovió un estudio científico que planteó como conclusión el contexto favorable que se creaba una vez visionada la serie contribuyendo a enfrentar sentimientos depresivos o de suicidio de una forma más favorable (Lauricella, Cingel y Wartella, 2018). Sin embargo, no toda la comunidad académica coincidió con estas conclusiones.

Estudios, en el contexto de la psiquiatría, señalaron que la serie mantiene relación con el empeoramiento del estado de ánimo estableciendo la asociación entre ver la serie y el cambio en el estado de ánimo depresivo entre los adolescentes. Se experimentó un empeoramiento de su estado de ánimo después de ver la serie (Santana da Rosa y otros, 2019). Netflix, en 2019, realizó un nuevo montaje del capítulo suavizando la escena del suicidio y eliminando la original. Esta modificación surgió por la recomendación de expertos y de la American Foundation for Suicide Prevention que reportaba: «desde el estreno de la serie los suicidios en EE. UU. en la franja de edad de 10 a 17 años aumentaron un 28,9\%» (Netflix suaviza en un remontaje la secuencia del suicidio de 'Por trece razones', 2019); (Bridge, y otros, 2019; Zimerman, y otros, 2018; Campo y Bridge, 2018).

Los estudios de la primera temporada de 13 Reasons Why demuestran «que la narrativa transmedia permite una aproximación más completa a temas sensibles, a partir de la interacción con la audiencia» (Atarama-Rojas y Requena Zapata, 2018, p. 193). Los productores de la serie incorporaron las redes sociales para promoverla y que los seguidores interactuaran sobre las cuestiones que planteaba. La segunda temporada, disponible desde el 18 de mayo de 2018, presagiaba aportar más detalles 
Pérez-Martínez, V. M., Aparicio Vinacua, B. y Rodríguez-González, M. D. Acoso escolar, violación y suicidio en twitter: segunda temporada de "por trece razones».

a la trama. A partir de un guion ya no vinculado directamente a la novela de Jay Asher profundizó en otros aspectos del relato que la primera temporada no abarcó.

\subsubsection{Segunda temporada}

En la segunda temporada, marco de esta investigación, el hilo conductor es el juicio contra el Liberty High School de los padres de Hannah Baker, Olivia Baker (Kate Walsh) y Andy Baker (Brian d'Arcy James). La acusación se centró en: las autoridades y los profesores del instituto eran conocedores del acoso que estaba teniendo Hannah Baker, de no tomar acciones al respecto $\mathrm{y}$, como consecuencia, eran responsables de su suicidio.

Las personas mencionadas por Hannah Baker en los casetes son citadas al juicio como testigos aportando una perspectiva personal de los hechos a partir de las preguntas del abogado de la familia Baker, Dennis Vásquez (Wilson Cruz), y de la defensa del instituto, Lainie Jensen (Amy Hargreaves). Sin embargo, los casetes no son pruebas en el caso porque se desconoce dónde están y la prueba fundamental son los hechos, las declaraciones de los testigos y otras pruebas que se van aportando en el transcurso del proceso.

Los testimonios de las personas que conocieron a Hannah Baker no son unánimes: van coincidiendo, ampliando más detalles, aportando otra perspectiva o contradiciendo la versión expuesta por Hannah en los casetes y que constituyeron el hilo conductor de la primera temporada. Se incorpora otra trama en la temporada. Clay Jensen vuelve a ser el receptor, en esta ocasión de fotografías tipo polaroid, de información que compromete a varios compañeros del colegio, a la propia Hannah Baker y a los integrantes del equipo de béisbol del Liberty High School.

La temporada avanza hasta el descubrimiento de cuestiones claves como: a) Hannah Baker fue expulsada de un colegio porque estaba en un grupo que acosaba a una niña (hecho por el cual se arrepintió); b) del comportamiento vejatorio y de violaciones a chicas por parte de algunos jugadores del equipo de béisbol del Liberty High School; c) identificar a Bryce Walker como el violador de Hannah Baker y de otras chicas del instituto (Jessica Davis y Chloe Rice). La temporada culminó con un episodio abierto en el cual Tyler Down desea tomar venganza, utilizando un arma de fuego, contra un grupo de compañeros e integrantes del grupo de béisbol por la agresión sexual propinada por ellos en el baño del colegio.

\subsubsection{Percepción de las temporadas}

Los espectadores identificaron diferencias entre la primera y la segunda temporada. Los registros de búsquedas de la serie en Google así lo reflejan (ver Gráfico 1): el 9 de abril de 2017 (primera temporada) y 20 de mayo de 2018 (segunda temporada) son los picos de búsqueda más representativos. La primera temporada registró un mayor número de días de interés desde su estreno (31 de marzo de 2017) con respecto a la segunda temporada y su estreno (18 de mayo de 2018). 
Pérez-Martínez, V. M., Aparicio Vinacua, B. y Rodríguez-González, M. D. Acoso escolar, violación y suicidio en twitter: segunda temporada de "por trece razones».

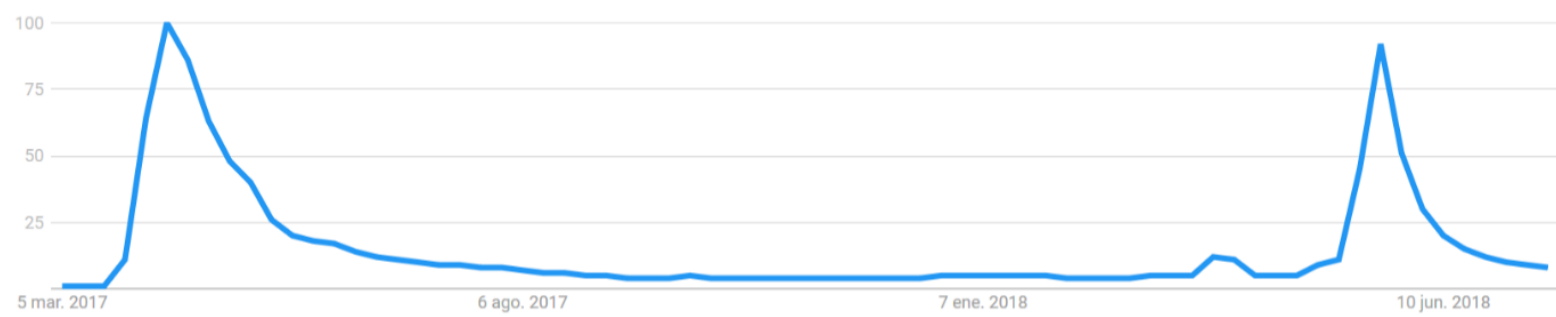

Gráfico 1. Registros de búsquedas en Google Trends. Criterios de búsqueda: a)

Región: todo el mundo; b) Fechas: 1/3/17 - 18/7/18; c) Categoría: Arte y entretenimiento; d) Búsqueda: en web; e) Fecha de la búsqueda: 19 de junio de 2019.

Fuente: Google Trends

Rotten Tomatoes (ver Imagen 1), le otorga a la primera temporada una valoración de la crítica del 79\% y de la audiencia un $80 \%$; con respecto a la segunda temporada la diferencia es significativa: 25\% (crítica) 52\% (audiencia). Metacritic le otorga a la primera temporada una puntuación de 64 sobre 100; a la segunda, 49 sobre 100.
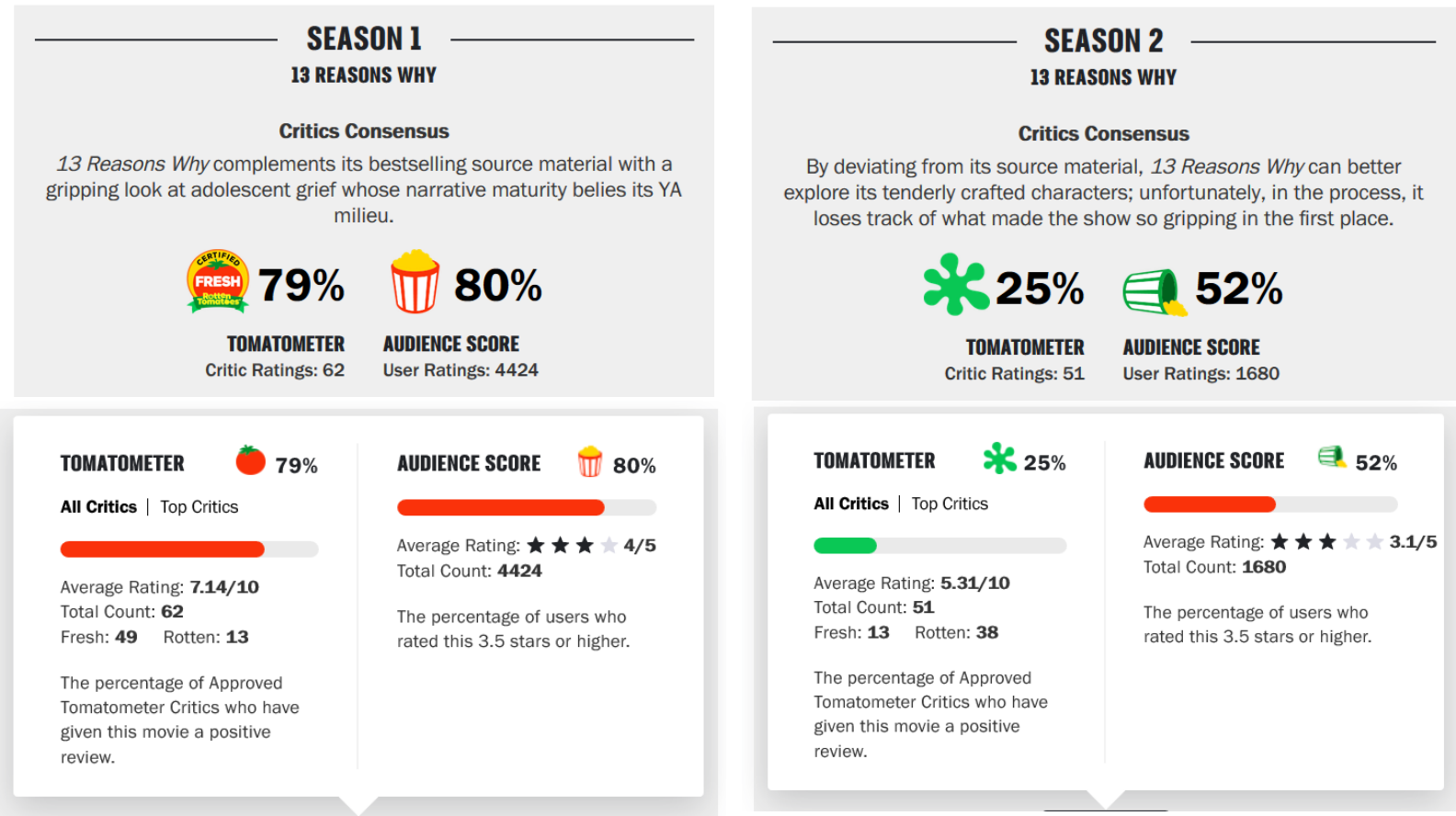

Imagen 1: Datos comparativos de la valoración de la serie en Rotten Tomatoes. Consulta realizada: 10 de julio de 2019.

Fuente: Rotten Tomatoes.

En Weighted Average Ratings (IMDb) la valoración general de las dos temporadas alcanzó un 8,0 (ver Gráfico 2). Sin embargo, la segunda temporada $(7,6)$ no alcanzó el nivel de aceptación que tuvo la primera $(8,4)$. El episodio (temporada 1) con la puntuación más baja fue el tercero con 7,9. En la segunda temporada un episodio 
Pérez-Martínez, V. M., Aparicio Vinacua, B. y Rodríguez-González, M. D. Acoso escolar, violación y suicidio en twitter: segunda temporada de «por trece razones».

obtuvo el mismo puntaje (episodio seis) y tres obtuvieron una valoración superior (episodios nueve, once y doce).

En un análisis longitudinal se puede considerar lo siguiente: ambas temporadas han mejorado las valoraciones mientras los episodios avanzaban. Es pertinente resaltar las valoraciones del último episodio: a) en la primera temporada fue el episodio mejor valorado dejando la serie en un punto de expectativa alto con respecto a la segunda temporada; b) en la segunda temporada el último episodio obtuvo la peor valoración; no solo en comparación con los episodios de la segunda temporada sino también con los de la primera temporada.

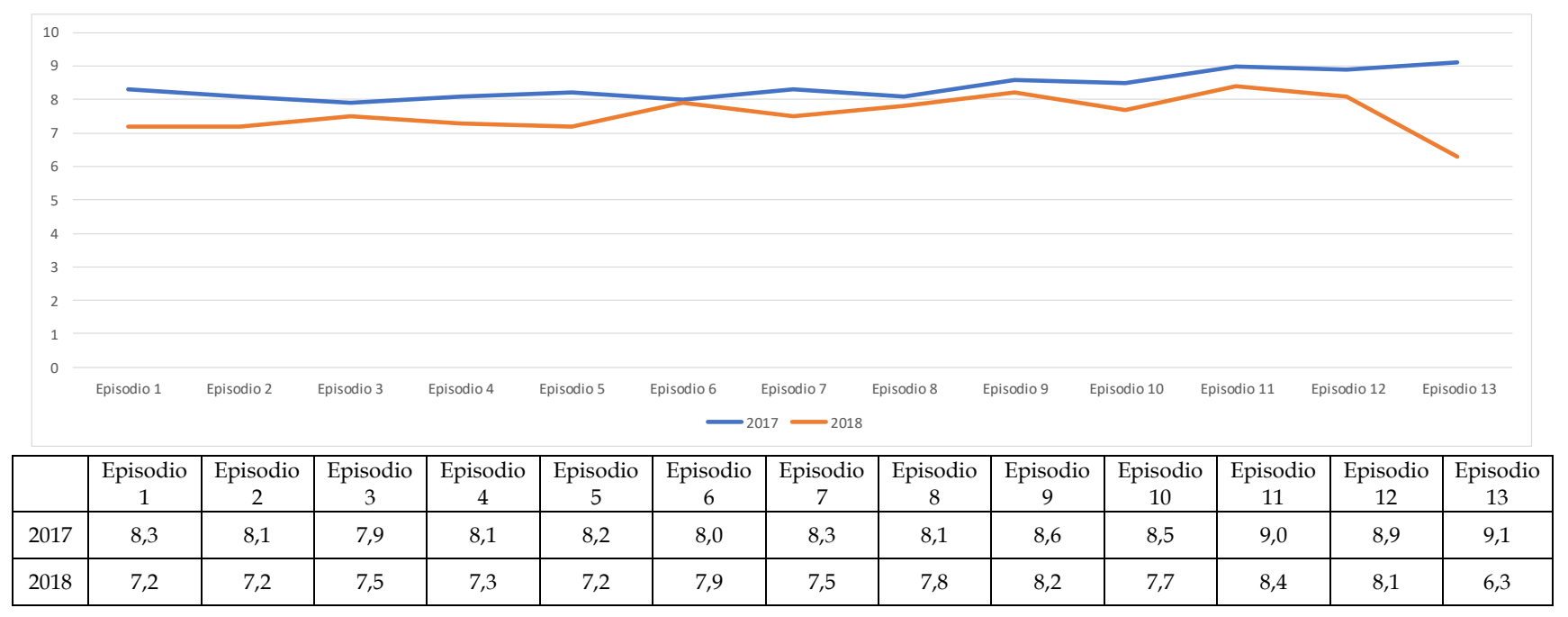

Gráfico 2: Comparativa de las valoraciones de los episodios de la primera y la segunda temporada. La guía para padres de IMDb valora las siguientes categorías: Sex \& Nudity (severo), Violence \& Gore (severo), Profanity (severo), Alcohol, Drugs \& Smoking (moderado), Frightening \& Intense Scenes (severo). Consulta realizada: 10 de julio de 2019.

Fuente: IMDb.

\section{OBJETIVOS}

Twitter es una red social de interés para el estudio sobre las audiencias (Deltell Escolar, Claes y Osteso López, 2013) o, como en el caso que nos ocupa, por su capacidad de interactividad entre sus usuarios (Lis Gindin, Castro Rojas, Coiutti, Cardoso y Rostagno, 2019). En este estudio optamos estratégicamente por Twitter por sus características: plataforma microblogging, enfoque abierto, contenidos simples, espontaneidad, posibilidad de interacción con otros usuarios, uso de recursos multimedia y, elemento clave, aporta «movilidad» (Blanco y Sueiro, 2014).

Las temáticas sobre el acoso escolar, la violación y el suicidio están presentes en todos los episodios de la primera y segunda temporada de 13 Reasons Why. En esta 
Pérez-Martínez, V. M., Aparicio Vinacua, B. y Rodríguez-González, M. D. Acoso escolar, violación y suicidio en twitter: segunda temporada de "por trece razones».

investigación nos concretamos en la segunda temporada. Las dos hipótesis de partida fueron:

H1. Las temáticas sobre el acoso escolar, la violación y el suicidio son debatidas en Twitter por seguidores de la segunda temporada de 13 Reasons Why. Expusieron y plantearon cuestiones relevantes sobre cómo se expusieron estos temas. Aportaron, además, sus percepciones creándose un entorno virtual de debate sobre estas cuestiones. Si la presencia y su interactividad en Twitter fueron cuantitativamente significativas se puede considerar que los temas sobre acoso escolar, violación y suicidio fueron de interés para la audiencia de la serie.

H2. Las redes sociales, y en el caso que nos ocupa Twitter, fomentan una interacción discursiva entorno a temas concretos relacionados con las series de televisión que contribuyen a la expansión del mensaje aportando una nueva dimensión a la transmedialidad de las narrativas. Esta interacción cobra relevancia cuando el usuario utiliza el etiquetado (hashtag) como método para recuperar contenidos o participar en la comunidad de usuarios interesados en estas temáticas.

Desde estas premisas, el objetivo general fue analizar, desde una perspectiva cuantitativa, los comentarios sobre el acoso escolar, la violación y el suicidio relacionados con la segunda temporada de 13 Reasons Why en Twitter. Como objetivos específicos nos planteamos: 1) identificar, desde una perspectiva cuantitativa, las características de los comentarios publicados; 2) determinar cómo la serie abordó las temáticas a partir de los comentarios publicados. Las preguntas de investigación fueron:

P1. Con motivo de la segunda temporada de 13 Reasons Why: en el contexto de un análisis cuantitativo ¿cuáles son las características de los comentarios publicados en Twitter sobre el acoso escolar, la violación y el suicidio?

P2. En relación con la presencia de estas temáticas en la serie: ¿características cuantitativas de las valoraciones realizadas sobre el enfoque de estos temas en la segunda temporada de 13 Reasons Why?

\section{METODOLOGÍA}

Las series para adolescentes cada vez tienen más presencia en plataformas de videos por demandas. Son varias las series que podrían haber sido seleccionadas en este estudio entre 2017 y 2019: Élite, Sex Education, Quicksand, The Society, Chilling Adventures of Sabrina, The End of the F**ing World, Baby, Insatiable, Everything Sucks!, entre otras.

13 Reasons Why reunía varias características que contribuyeron a su elección: a) la presencia de tramas y subtramas de interés para el público adolescente; b) el origen de la serie en la novela de Jay Asher, Por trece razones, adaptada para ser distribuida por Netflix; c) el éxito de audiencia que esta serie había logrado en su primera 
Pérez-Martínez, V. M., Aparicio Vinacua, B. y Rodríguez-González, M. D. Acoso escolar, violación y suicidio en twitter: segunda temporada de "por trece razones».

temporada (2017); d) los elementos narrativos que quedaron sin resolver en la primera temporada y los cuales tampoco tenía relación con la novela de Jay Asher; e) la participación activa de los fans en las redes sociales sobre las expectativas de la segunda temporada; f) la propuesta de la segunda temporada se focalizaba en profundizar en las explicaciones sobre los personajes que se vieron involucrados en la violación de Hannah Baker y la acusación contra el Liberty High School.

Las temporadas de 13 Reasons Why tienen su propio hilo narrativo. Son tres temporadas. Se visualizaron 26 episodios: la primera temporada (13 episodios) disponible desde el 31 de marzo de 2017 y la segunda temporada (13 episodios) desde el 18 de mayo de 2018. Se excluyó la tercera temporada porque se alejaba del hilo argumental de las dos anteriores.

Se utilizaron algunas de las características del análisis fílmico como herramienta de análisis. Concretamente la identificación de las escenas para obtener las tramas y subtramas de estas: entendiendo como escena «la unidad narrativa que consta de uno o varios planos con una continuidad espacial y/o temporal» (Soto, 2015, pág. 30). La revisión de los episodios se concentró en las tramas y subtramas. Se establecieron conexiones de los episodios de la segunda temporada con respecto a la primera para disponer de datos que explicaran algunos de los contenidos publicados en Twitter con motivo de la segunda temporada.

Una vez disponible, para su visualización, la segunda temporada de 13 Reasons Why se recuperaron los tuits. La muestra no fue probabilística; pero, sí fue estratégica (Igartua Perosanz, 2006). Se utilizaron filtros para seleccionar los tuits ajustados a los objetivos de la investigación y minimizando el sesgo en su recuperación. La plataforma para el stream y recuperación de los tuits fue Followthehashtag: herramienta testeada en otros estudios (Blasco-Duatis, Coenders Gallart y Sáez, 2018; Piñeiro-Otero y Martínez-Rolán, 2017; Pérez-Martínez, Rodríguez González y Tobajas Gracia, 2017; Pérez-Martínez, 2016).

\subsection{Marco muestras y submuestras}

Los criterios y filtros utilizados para seleccionar la muestra fueron:

1. Período de recuperación de tuits. Inicio: 17 de mayo de 2018 (desde las 03:48 horas). Final: 8 de enero de 2019 (hasta las 17:45 horas).

2. Se recuperaron los tuits con hashtags específicos. Los hashtags son «palabra clave» (Moreno, 2014) y una manera de etiquetar para organizar, recuperar y precisar sobre cuáles cuestiones escribir o crear interacciones con otros usuarios en Twitter. Se seleccionaron tuits que contuvieran el hashtag \#13Reasons Why (título original) y/o el hashtag \#PorTreceRazones (título con el cual se conoció la serie en España).

3. Se excluyen los retuits. 
Pérez-Martínez, V. M., Aparicio Vinacua, B. y Rodríguez-González, M. D. Acoso escolar, violación y suicidio en twitter: segunda temporada de "por trece razones».

4. En esta primera recuperación se recuperaron 154.470 tuits.

5. Se excluyeron los tuits duplicados: aquellos donde había coincidencia exacta del nickname y el contenido del tuit. Marco muestral: $\mathrm{N}=151.570$ tuits.

A partir del marco muestral ( $\mathrm{N}=151.570$ tuits) se conformaron submuestras. La primera submuestra $\left(\mathrm{n}_{1}\right)$ se configuró utilizando palabras clave (keywords) que estaban organizadas por categorías (ver Tabla 1 ). Tuits recuperados: $n_{1}=4.053$.

Tabla 1. Selección de tuits con palabras claves: suicidio, suicide, suicidio.

\begin{tabular}{|llll|}
\hline Acoso escolar & Violación & & Suicidio \\
\cline { 1 - 1 } $\begin{array}{l}\text { acoso, bullying, intimidation, } \\
\text { bullying, bullismo }\end{array}$ & $\begin{array}{l}\text { violación, raped, le viol, } \\
\text { estupr(ada), violazione }\end{array}$ & & $\begin{array}{l}\text { suicidio, suicide, suicide, } \\
\text { suicidio, suicidio }\end{array}$ \\
\hline $\begin{array}{l}\text { Nota. Las palabras clave se consideraron tomando en cuenta las siguientes lenguas: español, inglés, } \\
\text { francés, portugués e italiano. }\end{array}$ & & \\
\hline
\end{tabular}

Fuente: Elaboración propia.

A partir de la $\mathrm{n}_{1}$ se procedió a recuperar los tuits agrupándolos en tres categorías. Los criterios fueron: recuperar los 100 tuits con mayor impacto (impacto $=$ favoritos + retuits) por cada una de las categorías. Se utilizó el «muestreo con reposición»: una unidad de registro (tuit) podía estar vinculada a más de una categoría y el análisis se tenía que realizar en el marco de referencia de esas categorías. Al finalizar este proceso hubo categorías que sobrepasaron los 100 tuis y se amplió el número de unidades de análisis. Las submuestras por categorías fueron: acoso escolar $\left(\mathrm{n}_{2}=122\right)$, violación $\left(\mathrm{n}_{3}=124\right)$ y suicidio $\left(\mathrm{n}_{4}=330\right)$.

\subsection{Análisis de los tuits.}

El análisis de los tuits se concretó en: a) datos básicos y de identificación de los tuits; b) interactividad e impacto; c) contenido de los tuits. El libro de códigos contempló la definición de las categorías y variables (ver Tabla 2). Se procedió a la codificación inicial y análisis con el uso del software Statistical Package for the Social Sciences (SPSS).

Tabla 2. Libro de códigos para el análisis de los tuits.

\begin{tabular}{|c|c|c|}
\hline \multirow{5}{*}{$\begin{array}{l}\text { Datos de } \\
\text { identificación } \\
\text { del tuit. }\end{array}$} & $\begin{array}{l}\text { Fecha de } \\
\text { publicación. }\end{array}$ & Fecha en la que fue publicado el tuit. \\
\hline & $\begin{array}{l}\text { Hora de } \\
\text { publicación. }\end{array}$ & Hora en la que fue publicado el tuit. \\
\hline & Usuario (username). & $\begin{array}{l}\text { Corresponde al nombre utilizado en el perfil de la cuenta de } \\
\text { Twitter a la que pertenece el tuit. }\end{array}$ \\
\hline & $\begin{array}{l}\text { Cuenta (nickname o } \\
\text { nick) }\end{array}$ & Nombre que identifica al usuario precedido de “@” (*) \\
\hline & Tipo de cuenta. & $\begin{array}{l}\text { 1) Sí, está verificada por Twitter. 2) No está verificada por } \\
\text { Twitter. 3) Cuenta suspendida. 4) Cuenta protegida. 5) Se }\end{array}$ \\
\hline
\end{tabular}


Pérez-Martínez, V. M., Aparicio Vinacua, B. y Rodríguez-González, M. D. Acoso escolar, violación y suicidio en twitter: segunda temporada de "por trece razones».

\begin{tabular}{|c|c|c|}
\hline & & identificó cambio en el nickname. \\
\hline & País (country). & País que se incluye en el perfil de Twitter. \\
\hline & $\begin{array}{l}\text { Perfiles de las } \\
\text { cuentas. }\end{array}$ & $\begin{array}{l}\text { 1) 13ReasonsWhy: perfiles relacionados directamente con la } \\
\text { serie (están verificadas por Twitter). } \\
\text { 2) Netflix: perfil de la empresa (verificada por Twitter). } \\
\text { 3) Personales: cuentas de Twitter identificadas con nombres o } \\
\text { imágenes de personas. Se excluyen los profesionales del } \\
\text { campo de la comunicación social. } \\
\text { 4) Medios de comunicación. } \\
\text { 5) Gubernamentales/ públicas. } \\
\text { 6) No se identifica. } \\
\text { 7) Fans. } \\
\text { 8) ONG, asociaciones. } \\
\text { 9) Empresas (no medios de comunicación). }\end{array}$ \\
\hline & Favoritos (favorites). & Número de favoritos (like) del tuit. \\
\hline Interactividad & Retuits (retweets). & Número de retuits realizados al tuit. \\
\hline e impacto. & $\begin{array}{l}\text { Favoritos más } \\
\text { retuits }\end{array}$ & Suma del número de favoritos y de retuits (Favs+RTs). \\
\hline & Tuit (twet). & $\begin{array}{l}\text { Contenido del tuit. Se considera el tuit como unidad: incluye } \\
\text { los elementos multimedia e interactivos. }\end{array}$ \\
\hline $\begin{array}{l}\text { Contenidos de } \\
\text { los tuits. }\end{array}$ & Posicionamiento. & $\begin{array}{l}\text { 1) El comentario se circunscribe a la serie. 2) La serie NO } \\
\text { aborda adecuadamente el tema. 3) No se identifica el } \\
\text { posicionamiento. 4) La serie Sí contribuye a debatir sobre el } \\
\text { tema. 5) NO se relaciona con la serie. }\end{array}$ \\
\hline $\begin{array}{l}\left({ }^{*}\right) \text { En esta inv } \\
\text { Los resultado } \\
\text { «personal»o } \\
\text { identificable» }\end{array}$ & $\begin{array}{l}\text { igación mantendre } \\
\text { e la investigación } \\
\text { ssible» que pudier } \\
\text { no Alamán, 2015). }\end{array}$ & $\begin{array}{l}\text { s la confidencialidad de los perfiles y de los textos de los tuits. } \\
\text { bresentarán de manera agregada y no se incluirá ningún dato } \\
\text { ortar «información relativa a una persona física identificada o }\end{array}$ \\
\hline
\end{tabular}

Fuente: Elaboración propia.

\section{RESULTADOS Y DISCUSIÓN}

\subsection{Twitter como ámbito de debate sobre 13 Reasons Why}

Las unidades de registro obtenidas en la fase de recuperación de los tuits publicados permiten valorar positivamente el interés de los seguidores de la serie por compartir contenidos sobre la misma. La potencialidad de alcance de los 154.470 tuits recuperados es un reflejo de ese interés. La métrica lo evidencia (ver Tabla 3). Destacando también, con respecto al país de origen de los tuits que aporta esta información ( $\mathrm{n}=60.719)$, el interés por la serie y por las cuestiones planteadas en la misma. Sin embargo, el 73,1\% de los tuits están ubicados en diez países.

Era previsible, por el origen de la serie y la presencia de Netflix, que los Estados Unidos fuera el país con mayor porcentaje de tuits publicados $(27,7 \%)$, después seguirían: Francia $(11,5 \%)$, Reino Unido $(8,2 \%)$, Brasil $(8,1 \%)$, España $(6,4 \%)$, México $(3,2 \%)$, Italia $(3,0 \%)$, Canadá $(3,0)$, Filipinas $(2,0)$. El otro porcentaje $(26,9 \%)$ lo integran 91 países. 
Pérez-Martínez, V. M., Aparicio Vinacua, B. y Rodríguez-González, M. D. Acoso escolar, violación y suicidio en twitter: segunda temporada de "por trece razones».

Tabla 3. Sumario de la métrica de los tuits recuperados sobre 13 Reasons Why.

\begin{tabular}{|l|l|}
\hline Criterios de la búsqueda (keyword) & $\begin{array}{l}\text { \#13ReasonsWhy OR \#PorTreceRazones } \\
\text {-filter:nativeretweets -filter:retweets }\end{array}$ \\
\hline Inicio de la medición (measured data from) & $2018-05-17$ 03:48 \\
\hline Fin de la medición (measured data to) & $2019-01-08$ 17:45 \\
\hline Audiencia total (total audience) & 448.952 .578 \\
\hline Total de tuits (total tweets) & 154.470 \\
\hline Impresiones potenciales (total potential impressions) & 2.279 .896 .468 \\
\hline Media de tuits por hora (tweets per hour) & 27,20 \\
\hline Media de tuits por día (tweets per day) & 652,93 \\
\hline Cuentas indicando género (total gender detections) & 61.210 \\
\hline \% Cuentas indicando género (\% of detections) & $39.63 \%$ \\
\hline Cuentas indicando masculino (total male) & 25.863 \\
\hline Cuentas indicando femenino (total female) & 35.347 \\
\hline$\%$ Cuentas indicando masculino (\% Male) & $43 \%$ \\
\hline$\%$ Cuentas indicando femenino (\% Female) & $57 \%$ \\
\hline
\end{tabular}

Fuente: Elaboración propia.

\subsection{Acoso escolar, violación y suicidio}

El número de tuits recuperados para esta fase fueron 4.053 tuits: $66,25 \%$ ( $N=6.117$ tuits) publicados desde 3.568 cuentas de Twitter. La mayor frecuencia de publicación de tuits fue en el mes de mayo. Un resultado esperado al ser los días siguientes al estreno de todos los episodios de la serie el periodo de mayor interés para su visualización.

La distribución por categorías se conformó con 4.626 tuits. Se sumaron 521 tuits a la muestra inicial $(\mathrm{N}=4.053)$ : se identificó la presencia de keywords de categorías diferentes en un mismo tuit y se consideraron como unidades de registros. La distribución de los tuits, según la frecuencia de la categoría, permitió identificar la temática del suicidio $(57,8 \%)$ como el preferente: seguido de la violación $(32,9 \%)$ y el acoso escolar $(23,4 \%)$.

Con respecto a los países originarios de los tuits, entre las cuentas o perfiles que aportaban esta información ( $\mathrm{N}=1.806)$, el número de países fue amplio (80 países). No obstante, 10 países concentraron el 81,95\% de los tuits: Estados Unidos (40,09\%), Reino Unido (13,79\%), España (6,04\%), Canadá (4,71\%), Australia (4,37\%), Francia $(4,26 \%)$, India (2,55\%), Brasil (2,38\%), México (2,27\%), Sudáfrica (1,50\%) y los otros 70 países en su conjunto (18,05\%). La distribución por categorías (ver Tabla 4) permite valorar la preferencia de los temas en los tuits.

El suicidio fue el tema que tuvo mayor preferencia en comparación con las otras categorías. Estados Unidos y Reino Unido ocuparon, en las tres categorías, los dos primeros lugares respectivamente. España, se ubicó en quinto lugar en la temática del suicidio; pero, en acoso escolar y violación ocupó el tercer puesto. Canadá se posicionó en el tercer lugar de la categoría suicidio. 
Pérez-Martínez, V. M., Aparicio Vinacua, B. y Rodríguez-González, M. D. Acoso escolar, violación y suicidio en twitter: segunda temporada de «por trece razones».

Tabla 4. Distribución de las unidades de registro según categorías y país de la cuenta de Twitter.

\begin{tabular}{|c|c|c|c|c|c|c|c|c|}
\hline \multicolumn{3}{|c|}{ Acoso } & \multicolumn{3}{|c|}{ Violación } & \multicolumn{3}{|c|}{ Suicidio } \\
\hline País & $\mathrm{n}$ & $\%$ & País & $\mathrm{n}$ & $\%$ & País & $\mathrm{n}$ & $\%$ \\
\hline EEUU & 135 & 29,8 & EEUU & 221 & 41,1 & EEUU & 469 & 43,7 \\
\hline Reino Unido & 50 & 11,0 & Reino Unido & 101 & 18,8 & Reino Unido & 145 & 13,5 \\
\hline España & 48 & 10,6 & España & 26 & 4,8 & Canadá & 63 & 5,9 \\
\hline Brasil & 33 & 7,3 & Canadá & 25 & 4,6 & Francia & 59 & 5,5 \\
\hline Australia & 21 & 4,6 & Australia & 22 & 4,1 & España & 49 & 4,6 \\
\hline México & 20 & 4,4 & Irán & 12 & 2,2 & Australia & 47 & 4,4 \\
\hline Canadá & 13 & 2,9 & India & 11 & 2,0 & India & 26 & 2,4 \\
\hline Chile & 12 & 2,6 & Sudáfrica & 11 & 2,0 & México & 19 & 1,8 \\
\hline India & 12 & 2,6 & Francia & 10 & 1,9 & Sudáfrica & 14 & 1,3 \\
\hline Francia & 11 & 2,4 & México & 9 & 1,7 & Argentina & 13 & 1,2 \\
\hline Тор 10 & 355 & 78,4 & Top 10 & 448 & 83,3 & Top 10 & 904 & 84,2 \\
\hline Otros países & 98 & 21,6 & Otros países & 90 & 16,7 & Otros países & 170 & 15,8 \\
\hline Totales & 453 & 100 & Totales & 538 & 100 & Totales & 1074 & 100 \\
\hline
\end{tabular}

Fuente: Elaboración propia.

Los contenidos publicados en los tuits no siempre se limitaron a una categoría concreta. Se identificaron tuits que incorporaron otros temas (ver Tabla 5). Es complejo establecer una relación entre la participación de los usuarios con estas etiquetas o palabras clave y los datos estadísticos sobre el acoso escolar, las violaciones y el suicidio. Por otra parte, con respecto a estas categorías, no se pueden encasillar los tuits como pertenecientes exclusivamente a un único tema.

Tabla 5. Distribución y porcentajes de los tuits según su pertenencia a dos categorías.

\begin{tabular}{|c|c|c|c|c|c|c|}
\hline & Acoso & Suicidio & Violación & Acoso & Violación & Suicidio \\
\hline fr Tuits por categoría & 949 & 2343 & 1334 & 949 & 1334 & 2343 \\
\hline$\%(\mathrm{~N}=4053)$ & $23,41 \%$ & $57,81 \%$ & $32,91 \%$ & $23,41 \%$ & $32,91 \%$ & $57,81 \%$ \\
\hline $\begin{array}{l}\text { fr Tuits suma de las dos } \\
\text { categorías }\end{array}$ & \multicolumn{2}{|c|}{3292} & \multicolumn{2}{|c|}{2283} & \multicolumn{2}{|c|}{3677} \\
\hline fr Tuis con las dos categorías & \multicolumn{2}{|c|}{129} & \multicolumn{2}{|c|}{129} & \multicolumn{2}{|c|}{304} \\
\hline$\%(\mathrm{~N}=4053)$ & \multicolumn{2}{|c|}{$3,18 \%$} & \multicolumn{2}{|c|}{$3,18 \%$} & \multicolumn{2}{|c|}{$7,50 \%$} \\
\hline $\begin{array}{l}\text { \% con respecto a las } \\
\text { categorías }\end{array}$ & $13,59 \%$ & $5,51 \%$ & $9,67 \%$ & $13,59 \%$ & $22,79 \%$ & $12,97 \%$ \\
\hline $\begin{array}{l}\text { \% Tuits suma de las dos } \\
\text { categorías }\end{array}$ & \multicolumn{2}{|c|}{$3,92 \%$} & \multicolumn{2}{|c|}{$5,65 \%$} & \multicolumn{2}{|c|}{$8,27 \%$} \\
\hline
\end{tabular}

Fuente: Elaboración propia.

La presencia de más de una categoría en los tuis establece, al menos en una primera aproximación, algún tipo de relación entre ellas. En este marco la relación violación-suicidio es la más significativa por la frecuencia absoluta (304) y por la 
Pérez-Martínez, V. M., Aparicio Vinacua, B. y Rodríguez-González, M. D.

Acoso escolar, violación y suicidio en twitter: segunda temporada de "por trece razones».

frecuencia relativa en cada una de las categorías y en la muestra general. Las otras relaciones son también relevantes y concretamente la relación establecida entre violación-acoso. Relevante es también que 77 tuits $(\mathrm{N}=4053)$ incorporaran las tres categorías en sus contenidos.

\subsection{El debate en los tuits de mayor impacto}

La submuestra con los tuits de mayor impacto se concretó en 761 tuits pertenecientes a 682 cuentas de Twitter (ver Tabla 6). Estas cuentas, en su conjunto, permiten establecer el impacto potencial de los tuits publicados. Cuentas preferentemente de perfiles «personales» son las que generaron los tuits de mayor impacto: acoso $(77,1 \%)$, violación $(78,8 \%)$ y suicidio $(69,9 \%)$.

Las cuentas vinculadas a los tuits sobre el suicidio suman el mayor número de seguidores $(66,4 \%)$ y se puede considerar que el tema con mayor capacidad de impacto. No obstante, es también significativo el tema de la violación el cual ocupó el segundo grupo de tuits perteneciente a tuits que incluían esta categoría $(20,7 \%)$.

Tabla 6. Distribución de los tuis con mayor impacto por categorías.

\begin{tabular}{|c|c|c|c|c|}
\hline Categoría & Cuentas & Followers & Following & Listed \\
\hline Acoso & 194 & 8909603 & 207784 & 47457 \\
\hline Violación & 191 & 14313599 & 230541 & 91630 \\
\hline Suicidio & 405 & 45963364 & 1515848 & 326147 \\
\hline Totales: & $790\left(^{*}\right)$ & 69186566 & 1954173 & 465234 \\
\hline
\end{tabular}

Fuente: Elaboración propia.

Con respecto al engagement generado con los tuits se identifica un mayor interés hacia la categoría «acoso» tanto por los números de favoritos como los retuits (ver Tabla 7). Se refleja en la suma de ambos (12,6\%). El tema del suicidio sigue siendo la categoría con mayor engagement (83,8\%).

Tabla 7. Distribución de los tuis con mayor impacto por categorías.

\begin{tabular}{|c|c|c|c|c|}
\hline Categoría & $\mathrm{N}^{0}$ de tuits & Favoritos & Retuits & Fav + Ret \\
\hline Acoso & 205 & 1080 & 251 & 1331 \\
\hline Violación & 203 & 288 & 88 & 376 \\
\hline Suicidio & 438 & 6934 & 1898 & 8832 \\
\hline Totales: & 846 & 8302 & 2237 & 10539 \\
\hline
\end{tabular}

Fuente: Elaboración propia.

El origen de las cuentas (ver Tabla 8), aquellas que las han identificado (40), indican que España y Estados Unidos son los países con mayor número de tuits 
Pérez-Martínez, V. M., Aparicio Vinacua, B. y Rodríguez-González, M. D. Acoso escolar, violación y suicidio en twitter: segunda temporada de "por trece razones».

enviados. Relevante, la mayoría de tuit de España encabeza la lista vinculada al acoso. Coincide también en primer lugar con los Estados Unidos con respecto a la temática de la violación.

Tabla 8. Distribución de los tuis por países de las cuentas de Twitter y categorías.

\begin{tabular}{|c|c|c|c|c|c|c|}
\hline \multirow[b]{3}{*}{ España } & \multicolumn{2}{|c|}{ Acoso } & \multicolumn{2}{|c|}{ Violación } & \multicolumn{2}{|c|}{ Suicidio } \\
\hline & $\mathrm{fr}$ & $\%$ & $\mathrm{fr}$ & $\%$ & fr & $\%$ \\
\hline & 30 & 30,3 & 20 & 20,2 & 22 & 9,69 \\
\hline Estados Unidos & 17 & 17,2 & 20 & 20,2 & 78 & 34,36 \\
\hline Reino Unido & 10 & 10,1 & 14 & 14,1 & 28 & 12,33 \\
\hline México & 7 & 7,1 & 8 & 8,1 & 11 & 4,85 \\
\hline Chile & 4 & 4,0 & 6 & 6,1 & 6 & 2,64 \\
\hline Argentina & 4 & 4,0 & 5 & 5,1 & 5 & 2,20 \\
\hline Colombia & 1 & 1,0 & 5 & 5,1 & 3 & 1,32 \\
\hline Venezuela & 8 & 8,1 & 4 & 4,0 & 4 & 1,76 \\
\hline Perú & - & - & 3 & 3,0 & - & - \\
\hline Australia & 3 & 3,0 & 2 & 2,0 & 7 & 3,08 \\
\hline Canadá & - & - & 1 & 1,0 & 12 & 5,29 \\
\hline Alemania & - & - & 1 & 1,0 & 3 & 1,32 \\
\hline Ecuador & 1 & 1,0 & 1 & 1,0 & 1 & 0,44 \\
\hline Francia & 2 & 2,0 & 1 & 1,0 & 11 & 4,85 \\
\hline Guatemala & 1 & 1,0 & 1 & 1,0 & 1 & 0,44 \\
\hline India & - & - & 1 & 1,0 & 2 & 0,88 \\
\hline Italia & - & - & 1 & 1,0 & 3 & 1,32 \\
\hline Panamá & 1 & 1,0 & 1 & 1,0 & 1 & 0,44 \\
\hline Pakistán & - & - & 1 & 1,0 & 1 & 0,44 \\
\hline Uruguay & 2 & 2,0 & 1 & 1,0 & 2 & 0,88 \\
\hline Brasil & 4 & 4,0 & - & - & 3 & 1,32 \\
\hline Sudáfrica & 1 & 1,0 & - & - & 8 & 3,52 \\
\hline Finlandia & - & - & - & - & 2 & 0,88 \\
\hline Nueva Zelanda & - & - & - & - & 2 & 0,88 \\
\hline Otros países $\left.{ }^{*}\right)$ & 3 & 3,0 & 2 & 2,0 & 11 & 4,84 \\
\hline & 99 & 100 & 99 & 100 & 227 & 100 \\
\hline
\end{tabular}

Fuente: Elaboración propia.

El uso del inglés (ver Tabla 9) es preferente en las categorías de «violación» y «suicidio». Ahora bien, en lo referente al tema sobre el acoso, y quizás por el número superior de tuits enviados desde perfiles de España, el español tiene una amplia presencia en los tuits $(55,6 \%)$. 
Pérez-Martínez, V. M., Aparicio Vinacua, B. y Rodríguez-González, M. D. Acoso escolar, violación y suicidio en twitter: segunda temporada de "por trece razones».

Tabla 9. Distribución de los tuis por categoría y lenguaje utilizado en los tuits.

\begin{tabular}{|c|c|c|c|c|c|c|}
\hline \multirow[t]{2}{*}{ Lenguaje } & \multicolumn{2}{|c|}{ Acoso } & \multicolumn{2}{|c|}{ Violación } & \multicolumn{2}{|c|}{ Suicidio } \\
\hline & $\mathrm{fr}$ & $\%$ & fr & $\%$ & fr & $\%$ \\
\hline Inglés & 77 & 37,6 & 106 & 52,2 & 288 & 65,8 \\
\hline Español & 114 & 55,6 & 96 & 47,3 & 108 & 24,7 \\
\hline Portugués & 13 & 6,3 & - & - & 8 & 1,8 \\
\hline Turco & 1 & 0,5 & - & - & - & - \\
\hline Francés & - & - & 1 & 0,5 & 21 & 4,8 \\
\hline Italiano & - & - & - & - & 13 & 3 \\
\hline Totales: & 205 & $100 \%$ & 203 & $100 \%$ & 438 & $100 \%$ \\
\hline
\end{tabular}

Fuente: Elaboración propia.

\subsection{Posicionamiento}

La mayoría de los tuits indican, después del análisis, que la serie «sí contribuye a debatir» sobre el acoso, la violación y el suicidio (ver Tabla 10). Esta apreciación fue mayor en los contenidos relacionados con el acoso $(72,2 \%)$.

En los temas sobre la violación o el suicidio la mayoría también mantuvieron una postura positiva hacia las posibilidades de la serie para crear debates; pero, aquellos que plantean que la serie «no aborda adecuadamente» es significativo.

Si recalculamos los porcentajes de los datos, dejando únicamente las variables «sí contribuye»y «no aborda adecuadamente», los tuits que expresaron que «no aborda adecuadamente» reflejan que no hay una percepción concluyente sobre como la serie enfoca estos temas: acoso (23,7\%), violación $(41,3 \%)$ y el suicidio $(37,9 \%)$.

Tabla 10. Distribución de los tuis por posicionamiento sobre la conveniencia o no de la serie.

\begin{tabular}{|c|c|c|c|c|c|c|}
\hline & \multicolumn{2}{|c|}{ Acoso } & \multicolumn{2}{|c|}{ Violación } & \multicolumn{2}{|c|}{ Suicidio } \\
\hline & fr & $\%$ & $\mathrm{fr}$ & $\%$ & fr & $\%$ \\
\hline La serie sí contribuye a debatir sobre el tema & 148 & 72,2 & 108 & 53,2 & 229 & 52,3 \\
\hline La serie no aborda adecuadamente el tema & 46 & 22,4 & 76 & 37,4 & 140 & 32,0 \\
\hline El comentario se circunscribe a la serie & 6 & 2,9 & 16 & 7,9 & 46 & 10,5 \\
\hline No se identifica el posicionamiento & - & - & 3 & 1,5 & 18 & 4,1 \\
\hline No se relaciona con la serie & 5 & 2,4 & - & - & 5 & 1,1 \\
\hline Total & 205 & 100 & 203 & 100 & 438 & 100,0 \\
\hline
\end{tabular}

Fuente: Elaboración propia. 
Pérez-Martínez, V. M., Aparicio Vinacua, B. y Rodríguez-González, M. D.

Acoso escolar, violación y suicidio en twitter: segunda temporada de "por trece razones».

\section{CONCLUSIONES}

Las teen series son un formato audiovisual de interés para los adolescentes y jóvenes. Su propuesta narrativa con la integración de otras plataformas y la expansión a través de las redes sociales constituyen una fortaleza/oportunidad para estas producciones. La audiencia, y entre ella los fans de las series, utilizan los canales digitales para compartir y expresar sus percepciones, sensaciones y experiencias sobre las historias, las tramas planteadas y los personajes. 13 Reasons Why no fue una excepción; pero, si es factible que la temática del suicidio, en un contexto social como los Estados Unidos, con indicadores tan significativos de acoso escolar y de suicidios constituyeran un foco de atención especial en un público adolescente ávido de conocer y expresar sus percepciones sobre estos temas.

El «lenguaje total», planteado por Vallet (1977), se concretaba en que era un «lenguaje verbo - audio - visual» (p. 18). La evolución de la tecnología y de las narrativas han hecho más complejo ese «lenguaje total»: lenguaje - verbo - audio visual - interactivo - móvil - multiplataforma - otros. Nos permite mantener esta idea los resultados de la investigación. Se visualiza cómo Twitter fue válido como espacio de debate sobre los temas expuestos en 13 Reasons Why. Tenemos que decodificar una mayor gama de códigos, los cuales, en función de la intencionalidad del emisor o emisores, puede tener una enmarañada diversidad de combinaciones.

En esta línea se evidencia la necesidad de formar a los jóvenes en la lectura y comprensión de los nuevos códigos. Aunque, esto no es novedoso. Se ha insistido en la incorporación en la enseñanza, desde la más temprana edad, de temas vinculados a las narrativas audiovisuales. Esta investigación corrobora también esa necesidad desde la visión más amplia del uso de múltiples canales digitales y online por parte de los jóvenes.

Sobre las dos primeras temporadas de 13 Reasons Why. No tuvieron la misma aceptación por parte de sus fans. Los datos indican que la segunda temporada no alcanzó el nivel de aceptación de la primera temporada e incluso su último episodio fue el peor valorado por un sector de su audiencia. La incoherencia de la trama, subtramas y personajes de la serie es significativa entre la primera y la segunda temporada dejando descolocada a la audiencia por las contradicciones en el relato, el cuestionamiento a las causas que motivaron el suicidio de Hannah Baker, la ausencia de una justicia eficaz por los casos de violación y las responsabilidades de las personas involucradas diluidas por los falsos testimonios o la incorporación de una segunda trama -el comportamiento de los jugadores de béisbol del instituto- que no encajaba en un relato coherente y mejor estructurado que sí estaba presente en la primera temporada.

Otra causa de la diferencia entre ambas temporadas apunta a las fortalezas de la primera temporada: la novedad de la serie, su relación con la novela de Jay Asher, las polémicas originadas por el enfoque de temas complejos, la forma como se expuso el suicidio de Hannah Baker. Sin embargo, la previsión indicaba que el interés hacia la 
Pérez-Martínez, V. M., Aparicio Vinacua, B. y Rodríguez-González, M. D. Acoso escolar, violación y suicidio en twitter: segunda temporada de «por trece razones».

segunda temporada estaba garantizado; pero, el desarrollo de esta no cumplió con las expectativas de la audiencia.

La hipótesis partida se corrobora. Las temáticas sobre el acoso escolar, la violación y el suicidio son debatidas en Twitter por seguidores de la segunda temporada de 13 Reasons Why. Al respecto, los autores de los tuits expusieron y plantearon cuestiones relevantes aportando, además, sus percepciones sobre cómo se expusieron estas cuestiones en la serie.

13 Reason Why se une a otras series (Everybody Hates Chris, Glee, The Simpsons, American Crime, Buffy the Vampire Slayer, Stranger Things, Big Little Lies, Degrassi, One Tree Hill, entre otras) que han abordado de manera directa o indirecta los temas que afectan a los adolescentes en un momento vital y complejo desde su visión personal y los cuales deben encajar en un entorno adolescente y juvenil. La segunda temporada de 13 Reason Why generó intercambio de información en aquellos temas centrales de la serie. Temas como la violación, el suicidio y el acoso estuvieron presentes en los contenidos publicados por su audiencia en Twitter etiquetando los tuits con la intención de comentar expresamente la serie y que sus mensajes fueran recuperados por otros usuarios de esta red social.

La interactividad entre los usuarios fue potencialmente importante por el nivel de engagement que registraron las cuentas de la muestra estudiada. Como se puede evidenciar también en otros estudios, Twitter, sigue siendo un canal válido para interactuar e intercambiar la experiencia de los fans sobre sus series de televisión. En el intercambio de tuits sobre la segunda temporada se crearon hilos de conversación en Twitter con críticas hacia la serie, algunas de ellas válidas, pero las cuestiones centrales de los contenidos expuestos en las historias de los personajes tuvieron una presencia significativa: la violación, el suicidio y el acoso.

Estados Unidos es el entorno en la cual las historias de 13 Reasons Why discurren y como consecuencia son las cuentas en Twitter que con mayor frecuencia generaron contenidos. Ahora bien, al margen de ser la plataforma Netflix quien distribuye la serie en otros países y de la estrategia publicitaria realizada, las cuentas Twitter de otros países de América y de Europa también se unieron al debate sobre los temas expuestos en la serie. Es el reflejo de la potencialidad de una red social como Twitter de establecer canales de comunicación y debates de contenidos de interés en aquellos problemas comunes en las sociedades o en colectivos concretos.

El estudio pretendía identificar, cuantitativamente, cómo la audiencia 13 Reasons Why planteaba en Twitter contenidos relacionados con el acoso escolar, la violación y el suicidio. Efectivamente, los resultados confirman esa presencia. La muestra elegida, en general y después de reorganizarla aplicando diferentes criterios, expone el interés de los seguidores de la serie por debatir sobre los temas del acoso, la violación y el suicidio. En ocasiones con contenidos que establecían relaciones entre estos temas. El debate estuvo presente en diferentes países generando un engagement significativo. El interés por el acoso escolar, la violación y el suicidio estuvo por 
Pérez-Martínez, V. M., Aparicio Vinacua, B. y Rodríguez-González, M. D.

Acoso escolar, violación y suicidio en twitter: segunda temporada de "por trece razones».

encima de la valoración que la audiencia tenía sobre la serie como propuesta audiovisual.

En relación cómo la serie expone los temas acoso escolar, la violación y el suicidio los resultados no son concluyentes. Aunque la mayoría exponen que la serie enfocó adecuadamente los temas hay un porcentaje significativo que indican que no; con mayor énfasis en el tema de la violación y el suicidio. No podemos aportar conclusiones más consolidadas al respecto. Sin embargo, Twitter es un canal válido para manifestar el apoyo o las divergencias sobre producciones como 13 Reasons Why y generar debates de interés para los fans y la sociedad.

\section{REFERENCIAS}

Abreu Sancho, V. A. \& Huamán Cruz, E. (2018). Análisis jurídico de la serie "13 reasons why". Persona y Familia: Revista del Instituto de la Familia, 1(7), 225-233. doi: $\underline{10.33539 / \text { peryfa.2018.n7.1259 }}$

Aguaded Gómez, J. I. (2005). Enseñar a ver la televisión: una apuesta necesaria y posible. Comunicar. Revista Científica de Educomunicación, 13(25), 51-55. doi: 10.3916/C25-2005-007

Aierbe-Barandiaran, A. \& Oregui-González, E. (2016). Valores y emociones en narraciones audiovisuales de ficción infantil. Comunicar. Revista Científica de Educomunicación, 24(47), 69-77. doi: 10.3916/C47-2016-07

Asher, J. (2007). 13 Reasons Why. USA: Razorbill.

Atarama-Rojas, T. \& Requena Zapata, S. (2018). Narrativa transmedia: análisis de la participación de la audiencia en la serie 13 Reasons Why para la aproximación al tema del suicidio. Fonseca, Journal of Communication, (17), 193-213. doi: $\underline{10.14201 / \text { fjc201817193213 }}$

Aubrey, J. S., Behm-Morawitz, E. \& Kim, K. (2014a). Understanding the effects of MTV's 16 and pregnant on adolescent girls' beliefs, attitudes, and behavioral intentions toward teen pregnancy. Journal of Health Communication, 19(10), 11451160. doi: $\underline{10.1080 / 10810730.2013 .872721}$

Aubrey, J. S., Behm-Morawitz, E. \& Kim, K. (2014b). Understanding the Effects of MTV' s 16 and Pregnant on Adolescent Girls 'Beliefs , Attitudes, and Behavioral Intentions Toward Teen Pregnancy. Journal of Health Communication, (19), 11451160. doi: $\underline{10.1080 / 10810730.2013 .872721}$

Becattini, E. (2018). When We Were Young. Netflix and the Teen Audience. Comunicazioni sociali, 40(2), 207-217. doi: $\underline{10.26350 / 001200000016}$ 
Pérez-Martínez, V. M., Aparicio Vinacua, B. y Rodríguez-González, M. D. Acoso escolar, violación y suicidio en twitter: segunda temporada de "por trece razones».

Benassini Félix, C. (2018). Contribución de las redes sociales a la transmedialidad de las teleseries. Global Media Journal México, 15(29), 202-2016. Recuperado de https://www.redalyc.org/jatsRepo/687/68758895011/html/index.html

Bertolli Filho, C. \& Pontalti Monari, A. C. (2018). "13 Reasons Why": o debate sobre o suicídio à tona na mídia brasileira. Revista Pauta Geral-Estudos em Jornalismo, 5(1), 1-18. Recuperado

http://www.revistas2.uepg.br/index.php/pauta/article/download/10677/20920 $\underline{9210015}$

Blanco, M. \& Sueiro, R. (2014). Cómo conseguir 10.000 seguidores en Twitter. Madrid: ESIC.

Blasco-Duatis, M., Coenders Gallart, G. \& Sáez, M. (2018). Representación composicional de la intermedia agenda-setting de los principales grupos de medios y partidos políticos en las elecciones generales españolas de 2015. Revista Latina de Comunicacion Social, (73), 264-292. doi: 10.4185/RLCS-2018-1255

Böttger, T., Cuadrado, F., Tyson, G., Castro, I. \& Uhlig, S. (2018). Open Connect Everywhere: A Glimpse at the Internet Ecosystem through the Lens of the Netflix CDN. ACM SIGCOMM Computer Communications Review, 48(1). Recuperado de http://qmro.qmul.ac.uk/xmlui/handle/123456789/36411

Bridge, J. A., Greenhouse, J. B., Ruch, D., Stevens, J., Ackerman, J., Sheftall, A. H., . . Campo, J. V. (2019). Association Between the Release of Netflix's 13 Reasons Why and Suicide Rates in the United States: An Interrupted Times Series Analysis [Article in Press]. Journal of the American Academy of Child \& Adolescent Psychiatry, 59(2), 236-243. doi: $10.1016 /$ j.jaac. 2019.04 .020

Cambra Badii, I. \& Mastandrea, P. (2017). Por 13 Razones: desafíos éticos frente al suicidio en una serie televisiva. Ética $\mathcal{E}$ Cine, 7(2), 37-45. http://hdl.handle.net/11336/49090

Campo, J. V. \& Bridge, J. A. (2018). Exploring the Impact of 13 Reasons Why: Looking for Light Amidst the Heat ... Journal of the American Academy of Child \& Adolescent Psychiatry, 57(8), 547-549. doi: 10.1016/j.jaac.2018.05.012

Carmichael, V. \& Whitley, R. (2018). Suicide portrayal in the Canadian media: examining newspaper coverage of the popular Netflix series '13 Reasons Why'. BMC Public Health, 1-10. doi: 10.1186/s12889-018-5987-3

Carrión Domínguez, Á. (2019). La Quality TV y la edad de oro de las ficciones seriadas. Zer, 24(46), 111-128. doi: $10.1387 /$ zer.20386 
Pérez-Martínez, V. M., Aparicio Vinacua, B. y Rodríguez-González, M. D. Acoso escolar, violación y suicidio en twitter: segunda temporada de "por trece razones».

Cortés Gómez, S., Martínez Borda, R. \& De la Fuente Prieto, J. (2016). Contribución de las redes sociales a la creación de narrativas transmedia a partir de las series de ficción en televisión. Comunicación y Hombre: Revista Interdisciplinar de Ciencias de La Comunicación y Humanidades, 12, 153-176. Recuperado de https://comunicacionyhombre.com/article/contribucion-las-redes-sociales-lacreacion-narrativas-transmedia-partir-las-series-ficcion-television/

Van Damme, E. \& Van Bauwel, S. (2010). 'I don't wanna be anything other than me': A case study on gender representations of teenagers in American teen drama series One Tree Hill. Interactions: Studies in Communication $\mathcal{E}$ Culture, 2(1), 17-33. doi: $\underline{10.1386 / \text { iscc.2.1.17_1 }}$

Damme, E. Van. (2010). Gender and sexual scripts in popular US teen series: A study on the gendered discourses in One Tree Hill and Gossip Girl. Catalan Journal of Communication $\mathcal{E}$ Cultural Studies, 2(1), 77-92. doi: 10.1386/cjcs.2.1.77_1

Del Moral Pérez, M. E. \& Villalustre Martínez, L. (2015). Mujeres mayores asturianas: visión crítica sobre la mediación televisiva e infancia. Píxel-Bit. Revista de Medios y Educación, (46), 167-186. doi: https:// www.researchgate.net/publication/273338799

Deltell Escolar, L., Claes, F. \& Osteso López, J. M. (2013). Audiencias televisivas y líderes de opinión en Twitter. Caso de estudio: El Barco. Estudios sobre el Mensaje Periodístico, 19(1), 347-364. doi: 10.5209/rev_ESMP.2013.v19.n1.42526

Donstrup, M. (2019). Género y poder en la ficción televisiva: análisis textual ideológico de una serie histórica. Doxa Comunicación, (28), 97-109. doi: 10.31921/doxacom.n28a05

Escalas, M. I. (2017). Transmedialidad y contenidos generados por los usuarios en la ficción española contemporánea: el caso de Vis a Vis (Atresmedia, 2015-2016) Sphera Publica, 2(17), 2-18. Recuperado de http://sphera.ucam.edu/index.php/sphera-01/article/view/326

Establés Heras, M. J. (2016). Entre fans anda el juego: audiencias creativas, series de televisión y narrativas transmedia. Opción (Especial, 11), 476-497. Recuperado de https://repositori.upf.edu/bitstream/handle/10230/33260/Estables_opc_entr.pd $\underline{\mathrm{f} \text { ? sequence }=1 \& \text { is Allowed }=\mathrm{y}}$

Feasey, R. (2006). Charmed: Why Teen Television. Journal of Popular Film and Television, 34(1), 2-9. doi: 10.3200/JPFT.34.1.2-9

Feijoo, B. \& Pavez, I. (2019). Contenido audiovisual con intención publicitaria en vídeos infantiles en YouTube: el caso de la serie Soy Luna. Communication $\mathcal{E}$ Society, 32(1), 313-331. doi: 10.15581/003.32.1.313-331 
Pérez-Martínez, V. M., Aparicio Vinacua, B. y Rodríguez-González, M. D. Acoso escolar, violación y suicidio en twitter: segunda temporada de "por trece razones».

Figuera-Maz, M., Tortajada, I. \& Araüna, N. (2008). La erótica del "malote". Lecturas adolescentes de las series televisivas: Atracción, deseo y relaciones sexuales y afectivas. Revista de Estudios de Juventud, (106), 49-61.

Fradegradi, M. (2016). Teen Wolf. Adolescenza e licantropia. Temi e motivi. Brumal. Revista de Investigación sobre lo Fantástico, 4(1), 79-106. doi: $10.5565 / \mathrm{rev} / \mathrm{brumal} .288$

Fuenmayor, V. \& García Aranguren, E. (2018). Los millennials y su reflejo en pantalla. Comunicación, (183-184), 59-65. Recuperado de https://comunicacion.gumilla.org/2019/02/21/los-millennials-y-su-reflejo-enpantalla/

Fuente Prieto, J., Lacasa Díaz, P. \& Martínez-Borda, R. (2019). Adolescentes, redes sociales y universos transmedia: la alfabetización mediática en contextos participativos. Revista Latina de Comunicación Social, 74, 172-196. doi: $\underline{10.4185 / R L C S-2019-1326}$

Gangopadhyay, S. \& Dhar, D. (2014). Social networking sites and privacy issues concerning youths. Global Media Journal-Indian Edition, 5(1), 1-7.

García García, P. J. (2011). Lo geek vende . Transformaciones de los topoi sobre el adolescente inadaptado en las series de televisión norteamericanas. Frame, (7), 159-190. Recuperado

https:// dialnet.unirioja.es/servlet/articulo?codigo $=3616638$

García Pastor, E. M. (2018). El Ministerio del Tiempo: la "propagabilidad" de una nueva forma de crear y consumir televisión en España. En (Ori, 2018), Nuevos horizontes de la literatura comparada (Vol. 1): Comparatismo digital (págs. 68-76). España: Sociedad Española de Literatura General y Comparada.

García-Muñoz, N. \& Fedele, M. (2011). Las series televisivas juveniles: tramas y conflictos en una «teen series». Comunicar. Revista Científica de Educomunicación, 19(37), 133-140. doi: 10.3916/C37-2011-03-05

García-Noblejas, J. J. (2017). Practical philosophy and television drama. Ethical and anthropological remarks on some European television series (2015). Church, Communication and Culture, 2(1), 41-62. doi: 10.1080/23753234.2017.1287279

Gerrad, Y. (2017). "It's a secret thing": Digital disembedding through online teen drama fandom. First Monday, 22(8), 1-15. doi: $10.5210 / \mathrm{fm} . v 22 \mathrm{i} 18.7877$

Gómez Rubio, Leire. y López Vidaldes, N. (2015). Del éxito en televisión a la participación en las redes sociales. Doxa. Comunicación, 20, 137-160.

Guarinos, V. (2009). Fenómenos televisivos «teenagers»: Prototipias adolescentes en series vistas en España. Comunicar, 16(33), 203-211. doi: 10.3916/c33-2009-03-012

Vivat Academia. Revista de Comunicación. 15 diciembre 2020 /15 marzo 2021, n 153, 137-168 
Pérez-Martínez, V. M., Aparicio Vinacua, B. y Rodríguez-González, M. D. Acoso escolar, violación y suicidio en twitter: segunda temporada de «por trece razones».

Guerrero-Pico, M. y Scolari, C. A. (2016). Narrativas transmedia y contenidos generados por los usuarios: el caso de los crossovers. Cuadernos.info, (38), 183-200. doi: $\underline{10.7764 / \text { cdi. } 38.760}$

Igartua Perosanz, J. J. (2006). Métodos cuantitativos de investigación en comunicación. Barcelona: Bosch.

Jenkins, H. (2010). Transmedia Storytelling and Entertainment: An annotated syllabus. Continuum, 24(6), 943-958. doi: 10.1080/10304312.2010.510599

Jenkins, H. (2010). Transmedia Generation [post]. Recuperado el 20 de marzo de 2019, de Confessions of an ACA-FAN: http://henryjenkins.org/blog/2010/03/transmedia_generation.html.

Josefa, M. \& Heras, E. (2016). Entre fans anda el juego: audiencias creativas, series de televisión y narrativas transmedia The Role of Fans as an Active and Creative Audience Through Transmedia Storytelling in TV Series. Especial, 32(11), 476-497.

Julián Gonzales, R. (2018). Nuevas representaciones en el ecosistema mediático: el documental transmedia como narrativa social. Estudio de caso "Proyecto Quipu". 10 Congresso Internacional Media Ecology and Image Studies: Desafios para as narrativas imagéticas. 684-697. Portugal: Ria. Disponible en: http://docs.wixstatic.com/ugd/43846c_9628362b9c4d4c938d2afe5de6a205c8.pdf.

Lacalle, C. \& Castro, D. (2018). Fandom televisivo y construcción de identidad. Análisis de los comentarios de las fans españolas y los community managers. Revista Latina de Comunicacion Social, (73), 1-18. doi:10.4185/RLCS-2018-1242

Lange, E. (2011). "We are all Gossip Girl": Gossip Girl and the Promise of Interactive Television. En (Pérez Gómez, 2011), Previously on: estudios interdisciplinarios sobre la ficción televisiva en la Tercera Edad de Oro de la Televisión. Sevilla: Biblioteca de la Facultad de Comunicación de la Universidad de Sevilla.

Lauricella, A. R., Cingel, D. P. \& Wartella, E. (2018). Exploring how teens and parents responded to 13 Reasons Why: Global Report. Northwestern University, Center on Media and Human Development, Evanston, IL.

Lis Gindin, I., Castro Rojas, S. R., Coiutti, N., Cardoso, A. L. \& Rostagno, J. F. (2019). Emoción en acción. El caso de \#RosarioSangra en Twitter (Rosario, Argentina, 2016). Ámbitos. Revista Internacional de Comunicación, 43(1), 48-69. doi: $\underline{10.12795 / \text { Ambitos.2019.i43.03 }}$

Llorent, V. J. \& Marín, V. (2013). La integración de los dibujos animados en el currículo de Educación Infantil. Una propuesta teórica. REICE. Revista Iberoamericana sobre Calidad, Eficacia y Cambio en Educación, 12(1), 73-82.

Vivat Academia. Revista de Comunicación. 15 diciembre 2020 / 15 marzo 2021, nº 153, 137-168 
Pérez-Martínez, V. M., Aparicio Vinacua, B. y Rodríguez-González, M. D. Acoso escolar, violación y suicidio en twitter: segunda temporada de «por trece razones».

López, S. R. \& Fernández, M. R. (2016). Las series de televisión en las redes sociales: Estudio de caso. Opción, 12, 525-540.

Morduchowicz, R. (. (2008). Los jóvenes y las pantallas. Nuevas formas de socablidad. Barcelona: Gedisa.

Moreno, M. (2014). El gran libro del community manager. $3^{a}$ ed. Barcelona: Gestión 2000.

Nabawy, G., Moawad, A., Gad, G. \& Ebrahem, S. (2016). The Relationship between use of Technology and Parent-Adolescents Social Relationship. Journal of Education and Practice, 7(14), 168-178.

Navarro-Abal, Y. \& Climent-Rodríguez, J. A. (2014). El efecto socializador del medio televisivo en jóvenes. Influencia de las conductas de gestión del conflicto mostradas por personajes de series de ficción. Área Abierta, 14(1), 25-42. doi: $\underline{10.5209 / \mathrm{rev} \_ \text {ARAB.2014.v35.n1.44684 }}$

Navarro-Abal, Y., Climent-Rodríguez, J. A. \& Fernández-Garrido, J. (2012). Modelos de gestión de conflictos en serie de ficción televisiva. Escritos de Psicología, 5(3), $52-$ 60. doi: $10.5231 /$ psy.writ.2012.0811

Netflix suaviza en un remontaje la secuencia del suicidio de 'Por trece razones'. (16 de julio de 2019). El País. Disponible en: https://elpais.com/cultura/2019/07/16/ television/1563269672_064978.html

Oliveira, S. F. \& Ribeiro, N. H. (2018). 13 Reasons Why: na perspectiva da gestão escolar/coordenação pedagógica. Pedagogia em Ação, 10, 26-38.

Osborne, P. (s.f.). Yorkey, Brian, creator, 13 Reasons Why. Netflix, 2017. . Gender Forum, 67, (2018): 103-106,111. Florida State University, USA.

Palin, E. (2009). Da Beverly Hills a 90210 : ricorrenze e variazioni nell'universo del teen drama. Comunicazioni social, 1, 69-79. doi: 10.1400/175314

Pano Alamán, A. (2015). Ironía verbal y actividad de imagen en el discurso de políticos y ciudadanos españoles en Twitter. Soprag, 3(1), 59-89.

Pasquier, D. (1997). Teen Series' Reception: Television, Adolescence and Culture of Feelings. Childhood: A Global Journal of Child Research, 351-373.

Pérez-Martínez, V. M. (2016). Las representaciones de la familia en Twitter: una panorámica desde la perspectiva de las virtudes sociales [The representations of family in Twitter: an overview from the perspective of social virtues]. Church, Communication and Culture, 1(1), 47-72. doi: $10.1080 / 23753234.2016 .1181303$ 
Pérez-Martínez, V. M., Aparicio Vinacua, B. y Rodríguez-González, M. D. Acoso escolar, violación y suicidio en twitter: segunda temporada de "por trece razones».

Pérez-Martínez, V. M., Rodríguez González, M. D. \& Tobajas Gracias, M. (2017). Mobilization and political participation on Twitter . Case study of the hashtag \# SuperTuesday in the primaries of the presidential elections of the USA 2016. Revista Latina de Comunicación Social, (72), 679-703. doi: 10.4185/RLCS-2017-1186

Piñeiro-Otero, T. \& Martínez-Rolán, X. (2017). Los memes en el activismo feminista en la Red. \#ViajoSola como ejemplo de movilización transnacional. Cuadernos.Info, (39), 17-37. doi: $10.7764 /$ cdi.39.1040

Prawiyadi, L., Irawan Aritonang, A. \& Wijayanti, C. (2018). Analisis isi pesan bullying dalam serial Netflix "13 Reasons Why". Jurnal E-Komunikasi, 6(2), 1-12. Disponible en http://publication.petra.ac.id/index.php/ilmu-komunikasi/ article/view/8303/7497.

Puyosa, I. (2017). Bots políticos en Twitter en la campaña presidencial \#Ecuador2017. Contratexto, (27), 39-60. doi: 10.26439/contratexto.2017.027.002

Raya, I., Sanchez-Labella, I. \& Duran, V. (2018). The construction of the teenager profile on Netflix Tv Shows 13 Reasons Why and Atypical. Comunicacion y Medios, 37(37), 131-143. doi: 10.5354/0719-1529.2018.48631

Renato Nardi, E. \& Feliciano Brigagão, L. R. (2018). 13 Reasons Why: uma análise filosófica a respeito do suicídio sob a ótica de Camus, Freud e Schopenhauer e sua abordagem pedagógica no Ensino Médio. Revista do NESEF Filosofia e Ensino, 7(1), 11-27. doi: $10.5380 /$ nesef.v7i1.62479

Rios, S. (2015). Joey Potter: Final Girl Next Door. Journal of Popular Film and Television, 43(3), 136-147. doi: $10.1080 / 01956051.2015 .1043232$

Rodríguez Illera, J. L., Martínez Olmo, F. \& Galván, C. (2019). Los relatos digitales personales y las redes sociales en adolescentes. E-Curriculum, 17(1), 10-27. doi: $\underline{10.23925 / 1809-3876.2019 v 17 i 1 p 10-27}$

Ryalls, E. D. (2016). Ambivalent aspirationalism in millennial postfeminist culture on Gossip Girl. Communication and Critical/Cultural Studies, 2(13), 198-213.

Sáiz Serrano, J. \& Parra Monserrat, D. (2017). Formación del profesorado de historia y ficción televisiva: el aprovechamiento didáctico de las series históricas en educación secundaria. Revista Electrónica Interuniversitaria de Formación del Profesorado, 20(2), 95-109. doi: 10.6018/ reifop.20.1.284931

Sánchez Escámez, A. \& Baena Sánchez, M. J. (2016). Influencia de la serie animada Shin-Chan sobre niños escolarizados en el ámbito rural. ENSAYOS, Revista de la Facultad de Educación de Albacete, 31(1), 89-103. 
Pérez-Martínez, V. M., Aparicio Vinacua, B. y Rodríguez-González, M. D. Acoso escolar, violación y suicidio en twitter: segunda temporada de "por trece razones».

Sánchez-Olmos, C. (2016). Del sofá a YouTube: estudio de género sobre la interacción en la red social en torno a las series de TV españolas. Communication $\mathcal{E}$ Society, 29(2), 117-132. doi: $10.15581 / 003.29 .2 . s p .117-132$

Santana da Rosa, G., Santos Andrades, G., Caye, A., Paz Hidalgo, M., Alves Braga de Oliveira, M. \& Pilz, L. K. (2019). Thirteen Reasons Why: The impact of suicide portrayal on adolescents' mental health. Journal of Psychiatric Research, 108, 2-6. doi: 10.1016/j.jpsychires.2018.10.018

Santandreu Aranda, A. (2015). Myss Mystic 2.0. The Vampire Diaries' Caroline Forbes and the American DreamWith and Without Fangs. Oceánide, 7, https://dialnet.unirioja.es/servlet/articulo?codigo $=6236592$

Scolari, C. A. (2018). Introduction: from Media Literacy to Transmedia Literacy. En (Scolari, 2018): Teens, media and collaborative cultures. Exploiting teens' transmedia skills in the classroom (págs. 12-21). Barcelona: Universitat Pompeu Fabra.

Scolari, C. A. \& Establés, M. J. (2017). El ministerio transmedia: Expansiones narrativas y culturas participativas. Palabra Clave, 20(4), 1008-1041. doi: 10.5294/pacla.2017.20.4.7

Sidorenko Bautista, P., Calvo Rubio, L. M. \& Cantero de Julián, J. I. (2018). Marketing y publicidad inmersiva: el formato $360^{\circ}$ y la realidad virtual en estrategias transmedia. Miguel Hernández Communication Journal, 9(1), 19-47. doi: $\underline{10.21134 / \mathrm{mhcj} . v 0 \mathrm{i} 9.227}$

Soto, J. A. (2015). Manual de Producción Audiovisual. Santiago: Pontificia Universidad Católica de Chile.

Sprada Barbosa, J., Mendes, G., Oliveira, M., Corrêa, M., Shimabukuro, N. \& Amorim, C. (2018). Séries e internet: até que ponto elas interferem na ideação suicida? In (Leal, et al., 2018) Actas do 12o Congresso Nacional de Psicologia da Saúde (pp. 467-474). Lisboa: ISPA - Instituto Universitário.

Tapia Frade, A. \& Martín Guerra, E. (2016). Neurociencia aplicada a la televisión: medición de la atención y la emoción de la serie "FOREVER" / Neuroscience applied to television: measuring attention and emotion in the audience of FOREVER series. Vivat Academia, 0(134), 69. doi: 10.15178/va.2016.134.69-82

Tortajada, I. \& Willem, C. (2019). Creación de significado online: recoger las voces de los y las fans de series televisivas. Empiria. Revista de Metodología de Ciencias Sociales, (42), 99-112. doi: 10.5944/empiria.42.2019.23252

Trillo-Domínguez, M., Del Moral, C. \& Sedeño-Valdellós, A. (2019). El clipmetraje como nuevo formato periodístico transmedia: el titular de los nuevos medios. En ( 
Pérez-Martínez, V. M., Aparicio Vinacua, B. y Rodríguez-González, M. D. Acoso escolar, violación y suicidio en twitter: segunda temporada de "por trece razones».

Sánchez-Mesa, 2019) Narrativas transmediales. La metamorfosis del relato en los nuevos medios digitales. Barcelona: Gedisa.

Tur-Viñes, V. \& Rodríguez Ferrándiz, R. (2014). Transmedialidad: series de ficción y redes sociales. El caso de Pulseras Rojas en el grupo oficial de Facebook (Antena 3. España). Cuadernos.Info, 34, 115-131. doi: $\underline{10.7764 / \text { cdi.34.549 }}$

Vallet, A. (1977). El lenguaje total. Zaragoza: Editorial Luis Vives.

Van Damme, E. (2010). Gender and sexual scripts in popular US teen series a study on the gendered discourses in One Tree Hill and Gossip Girl. Catalan journal of communication $\mathcal{E}$ cultural studies, 1(2), 77-92.

Vázquez-Herrero, J., González-Neira, A. \& Quintas-Froufe, N. (2019). La audiencia activa en la ficción transmedia: plataformas, interactividad y medición. Revista Latina de comunicación Social, (74). doi: 10.4185/RLCS-2019-1322

Whiteside, E., Hardin, M., Decarvalho, L. J., Carillo, N. M. \& Smith, A. N. (2013). “I Am Not a Cow": Challenging Narratives of Empowerment in Teen Girls Sports Fiction. Sociology of Sport Journal, (30), 415-434.

Wulandari, A., Shinta Hapsari, B., \& Bram, B. (2018). Communication failures in Netflix drama series "13 Reasons Why": a pragmatic analysis. International Journal of Humanity Studies, 1(2), 264-272. doi: 10.24071/ijhs.2018.010212

Zhang, L. \& Cassany, D. (2019). El fenómeno «danmu» y la participación mediática: Comprensión intercultural y aprendizaje de lenguas a través de «El Ministerio del Tiempo». Comunicar. Revista Científica de Educomunicación, XXVII(58), 19-29. doi: 10.3916/C58-2019-02

Zimerman, A., Caye, A., Zimerman, A., Salum, G. A., Passos, I. C. \& Kieling, C. (2018). Revisiting the Werther Effect in the 21st Century: Bullying and Suicidality Among Adolescents Who Watched 13 Reasons Why. Journal of the American Academy of Child \& Adolescent Psychiatry, 57(8), 610-613. doi: $\underline{10.1016 / j . j a a c .2018 .02 .019}$

\section{AUTOR/ES:}

\section{Víctor Manuel Pérez-Martínez}

Profesor contratado doctor acreditado en la Universidad San Jorge. Asignaturas: Ciberperiodismo, Documentación, Diseño y desarrollo de entornos y materiales TIC. Fue investigador principal de los grupos de investigación: «Cultura Digital: 
Pérez-Martínez, V. M., Aparicio Vinacua, B. y Rodríguez-González, M. D. Acoso escolar, violación y suicidio en twitter: segunda temporada de "por trece razones».

mediaciones y discursos» y «Cyberspace Working Group» (reconocidos por el Gobierno de Aragón). Licenciatura, especialización y maestría en Comunicación Social (Universidad Católica Andrés Bello, Venezuela).

vmperez@usj.es

Orcid ID: http:/ / orcid.org/0000-0001-9387-6738

Google Scholar: https:// scholar.google.es/citations?user=WiTFPF8AAAAJ\&hl=es

ResearchGate: https://www.researchgate.net/profile/Victor_Perez_Martinez

Scopus ID: 57195428306

Academia.edu: https://usj-es.academia.edu/VictorPerez

\section{María Dolores Rodríguez-González}

Ingeniera de Sistemas (UNEXPO, Venezuela). Estudiante del Máster Universitario en Didáctica de las Matemáticas en Educación Secundaria y Bachillerato (UNIR, España). Profesora en áreas de Informática, Aplicación de la Informática a la Educación, Formación Humana, Proyecto. Especialista e-learning. Diseño metodológico. Desarrolladora multimedia, transmedia y online. Elaboración contenidos TIC.

mariadrodriguezdp@gmail.com

Orcid ID: http:/ / orcid.org/0000-0003-3373-5516

\section{Beatriz Aparicio Vinacua}

Doctoranda en el Doctorado en Relaciones de Género y Estudios Feministas en la Universidad de Zaragoza. Graduada en Periodismo y Comunicación Audiovisual por la Universidad San Jorge. Máster en Estudios de Género con especialización en Investigaciones Feministas por la Universidad Complutense de Madrid. Proyecto final: representación de la violencia de género y sexual en la miniserie de HBO Big Little Lies. Máster Universitario en Marketing y Comunicación Corporativa por la Universidad San Jorge.

beatriz6av@gmail.com

ORCID: https:// orcid.org/0000-0001-7342-9455 